TRANSACTIONS OF THE

AMERICAN MATHEMATICAL SOCIETY

Volume 362, Number 3, March 2010, Pages 1371-1411

S 0002-9947(09)04908-3

Article electronically published on October 8, 2009

\title{
STRUCTURES HAVING O-MINIMAL OPEN CORE
}

\author{
ALFRED DOLICH, CHRIS MILLER, AND CHARLES STEINHORN
}

\begin{abstract}
The open core of an expansion of a dense linear order is its reduct, in the sense of definability, generated by the collection of all of its open definable sets. In this paper, expansions of dense linear orders that have o-minimal open core are investigated, with emphasis on expansions of densely ordered groups. The first main result establishes conditions under which an expansion of a densely ordered group has an o-minimal open core. Specifically, the following is proved:

Let $\mathfrak{R}$ be an expansion of a densely ordered group $(R,<, *)$ that

is definably complete and satisfies the uniform finiteness property.

Then the open core of $\mathfrak{R}$ is o-minimal.

Two examples of classes of structures that are not o-minimal yet have ominimal open core are discussed: dense pairs of o-minimal expansions of ordered groups, and expansions of o-minimal structures by generic predicates. In particular, such structures have open core interdefinable with the original o-minimal structure. These examples are differentiated by the existence of definable unary functions whose graphs are dense in the plane, a phenomenon that can occur in dense pairs but not in expansions by generic predicates. The property of having no dense graphs is examined and related to uniform finiteness, definable completeness, and having o-minimal open core.
\end{abstract}

\section{INTRODUCTION}

Throughout, $\mathfrak{R}$ denotes a fixed, but arbitrary, first-order expansion of a dense linear order $(R,<)$ without endpoints, in a language extending $\{<\}$. The structure $\mathfrak{R}$ is o-minimal if every (parametrically) definable unary (that is, contained in $R$ ) set is a finite union of points and open intervals. By combining the power of definability and the tools of model theory with the wealth of examples now known, o-minimality has provided a rich framework for what has been termed "tame topology", a wide-ranging generalization of semialgebraic geometry. See the monograph [10] and the survey paper [13] for general references. But the development of o-minimality was also strongly influenced by purely model-theoretic considerations; as examples, see [7, 19, 32, 34, 35. This paper is more in the spirit of the latter group of works than the former in that our focus is more on advancing the general model theory of ordered structures than in providing applications to other branches of mathematics.

Received by the editors January 7, 2008 .

2000 Mathematics Subject Classification. Primary 03C64; Secondary 06F20.

Key words and phrases. o-minimal, open core, ordered group, ordered field, uniform finiteness, definably complete, exchange property, definable Skolem functions, elimination of imaginaries, independence property, atomic model, dense pair, generic predicate, tame pair.

The second author was partially supported by NSF Grant DMS-9988855. The third author was partially supported by NSF Grant DMS-0070743. 
We have attempted to make the material accessible to a broad audience, the main requirement being familiarity with first-order definability in densely ordered structures. Throughout, we let DLO denote the theory of dense linear orders without endpoints, in the language $\{<\}$.

Researchers have sought extensions beyond the o-minimal framework that preserve good behavior. For example, one might require only that every definable unary set be a finite union of convex definable sets; see Macpherson, Marker, and Steinhorn 24 for some work in this direction. Here, we go in an orthogonal direction introduced in Miller and Speissegger [28, namely, that the open (in the natural order topology) definable sets are well behaved, in particular, that every open definable unary set is a finite union of open intervals. This loose notion is made precise via the following.

Definition. The open core of $\mathfrak{R}$, denoted by $\mathfrak{R}^{\circ}$, is the structure $(R,(U))$, where $U$ ranges over the open sets, of all arities, definable in $\mathfrak{R}$. For concreteness, we regard $\mathfrak{R}^{\circ}$ as a structure in a language consisting precisely of relation symbols for the nonempty open sets definable in $\mathfrak{R}$. Given a theory $T \supseteq$ DLO, we say that a theory $T^{\prime}$ is an open core of $T$ if for every $\mathfrak{M} \vDash T$ there exists $\mathfrak{M}^{\prime} \vDash T^{\prime}$ such that $\mathfrak{M}^{\circ}$ is interdefinable with $\mathfrak{M}^{\prime}$.

As the set $\left\{(x, y) \in R^{2}: x<y\right\}$ is open, $\mathfrak{R}^{\circ}$ is an expansion of $(R,<)$. If $\mathfrak{R}$ expands an ordered group, then the group operation is continuous, so its graph is definable in $\mathfrak{R}^{\circ}$. Similarly, if $\mathfrak{R}$ expands an ordered ring, then its multiplication is also definable in $\mathfrak{R}^{\circ}$. More generally, we shall see later that if all primitive relations and functions of $\mathfrak{R}$ are Boolean combinations of open sets, then $\mathfrak{R}$ is interdefinable with $\mathfrak{R}^{\circ}$.

In this paper, we investigate expansions of dense linear orders that have o-minimal open core, with emphasis on expansions of densely ordered groups. This program was begun in [28, but the focus there was on expansions of the real field $(\mathbb{R},+, \cdot)$, and proofs tended to rely on working over $\mathbb{R}$. Here, we proceed from a more model-theoretic perspective. We extend some of the results from [28] in two ways: we do not require working over the real field, and we examine several model-theoretic properties of structures having o-minimal open core that were not considered in 28].

Before going any further, we should point out that the definition of o-minimal makes sense for expansions of arbitrary linear orders. The study of such structures resolves into those of o-minimal expansions of dense linear orders (without endpoints) and o-minimal expansions of discrete linear orders, and the latter subject is demonstrably trivial; see Pillay and Steinhorn 36, 37, for details. Hence, throughout this paper, o-minimality always includes the requirement that the underlying order be dense.

If $\mathfrak{R}^{\circ}$ is o-minimal, then every open unary set definable in $\mathfrak{R}$ is a finite union of intervals. One of the main results of [28] is a partial converse: If $\mathfrak{R}$ is an expansion of $(\mathbb{R},<,+)$ that has a pole - that is, $\mathfrak{R}$ defines a homeomorphism between a bounded interval and an unbounded interval - and every open definable unary set is a finite union of intervals, then $\mathfrak{R}^{\circ}$ is o-minimal. The assumption that $\mathfrak{R}$ have a pole is superfluous (see Proposition 2.14 below) but in order to replace working over $\mathbb{R}$ we introduce some uniformity: We assume that, in every elementarily equivalent structure, every open definable unary set is a finite union of intervals. By a routine 
compactness argument, this is equivalent to: For every $m \in \mathbb{N}$ and definable $A \subseteq$ $R^{m} \times R$ there exists $N \in \mathbb{N}$ such that for all $x \in R^{m}$ the interior of the set $\{t \in$ $R:(x, t) \in A\}$ is a disjoint union of at most $N$ open intervals. It is convenient to replace this condition by two others, definable completeness and uniform finiteness, each of independent interest.

Definition. We say that $\mathfrak{R}$ is definably (Dedekind) complete - for short, $\mathfrak{R}$ has DC or $\mathfrak{R} \vDash D C$-if every definable unary set has both a supremum and an infimum in $R \cup\{ \pm \infty\}$. For a theory $T \supseteq \mathrm{DLO}$, we write $T \vDash \mathrm{DC}$ if every model of $T$ has DC.

It is easy to see that $\mathfrak{R} \vDash D C$ if and only if every open definable unary set is a disjoint union of open intervals. Hence, if $\mathfrak{R}^{\circ}$ is o-minimal, then $\mathfrak{R} \vDash \mathrm{DC}$. Of course, every expansion of the real line $(\mathbb{R},<)$ has DC. On the other hand, DC is strong enough to permit a substantial amount of elementary point-set topology to be developed just as if working over $(\mathbb{R},<)$, a theme elaborated upon in [25. Some facts proved there that are relevant to this paper, along with some further technical consequences, are collected in Section 2 .

The second property of interest has been studied extensively by model theorists, though often under other names.

Definition. We say that a structure $\mathfrak{M}$ satisfies uniform finiteness - for short, $\mathfrak{M}$ has UF or $\mathfrak{M} \vDash \mathrm{UF}$ - if for every $m, n \in \mathbb{N}$ and $A \subseteq M^{m} \times M^{n}$ definable in $\mathfrak{M}$ there exists $N \in \mathbb{N}$ such that for every $x \in M^{m}$ the set $\left\{y \in M^{n}:(x, y) \in A\right\}$ either is infinite or contains at most $N$ elements. An easy induction shows that it suffices to consider only the case $n=1$. For a theory $T$, we write $T \vDash \mathrm{UF}$ if every model of $T$ satisfies UF.

One of the most important early results about o-minimal structures is that they satisfy UF; see Knight, Pillay, and Steinhorn [19. We are now ready to state the main result of this paper.

Theorem A. If $\mathfrak{R} \vDash \mathrm{DC}+\mathrm{UF}$ and expands an ordered group, then $\mathfrak{R}^{\circ}$ is o-minimal.

By the Cell Decomposition Theorem, every set definable in an o-minimal structure is a Boolean combination of open definable sets. Hence, we have the following characterization (compare with [28, Theorem (b)]).

Corollary. An expansion of a densely ordered group is o-minimal if and only if it satisfies both $\mathrm{DC}$ and UF and is interdefinable with its open core.

The proof of Theorem A concludes in Section 3. We follow the overall strategy used in [28, but our tactics are different, as we have neither separability of the topology nor the Baire Category Theorem available. Nevertheless, the proof requires only basic topology and first-order definability theory. As a corollary of the proof, we obtain an analogue of the Baire Category Theorem (3.5).

Theorem A constitutes a major component of our attempt to extend the program begun in 28. Next, we outline some results toward our second goal: a more modeltheoretically oriented investigation.

By definition, DC and UF are first-order schema, so both are elementary properties; that is, they are preserved under elementary equivalence of structures. (N.B. This is not the same as saying that they determine elementary classes.) We shall 
see (2.5) that an expansion of a densely ordered group has both DC and UF if and only if every open unary set definable in any elementarily equivalent structure is a finite union of intervals. Hence, another way to state Theorem A is this:

Theorem $\mathbf{A}^{\prime}$. If $T$ is an extension of the theory of densely ordered groups such that every open unary set definable in any model of $T$ is a finite union of open intervals, then every model of $T$ has o-minimal open core.

We would like to have the seemingly stronger conclusion that $T$ has an open core $T^{\prime}$, preferably complete. We do not yet know if this is true, though it is for all presently-understood examples. There is a natural candidate for $T^{\prime}$, roughly, the theory of all $T$-definable open sets. We address this issue more thoroughly in Sections 4 through 6 .

Next, we recall another key model-theoretic notion.

Definition. The definable closure in $\mathfrak{R}$ of $S \subseteq R$, denoted by $\operatorname{dcl}_{\mathfrak{R}}(S)$, is the set of all $r \in R$ defined in $\mathfrak{R}$ using parameters from $S$. The subscript $\mathfrak{R}$ is often suppressed. We say that $\mathfrak{R}$ satisfies the exchange property - for short, $\mathfrak{R}$ has $\mathrm{EP}$ or $\mathfrak{R} \vDash \mathrm{EP}$-if $b \in \operatorname{dcl}(S \cup\{a\})$ for all $S \subseteq R$ and $a, b \in R$ such that $a \in$ $\operatorname{dcl}(S \cup\{b\}) \backslash \operatorname{dcl}(S)$. For a theory $T$, we write $T \vDash$ EP if every model of $T$ has EP.

To be precise, the definition is that of exchange with respect to dcl, as EP can be formulated with respect to any pregeometry. But dcl is the only pregeometry that we shall deal with in this paper, bearing always in mind that dcl coincides with acl (algebraic closure) in ordered structures. Every o-minimal structure satisfies EP ([35, 4.1] combined with [19, 0.2]). Theories that satisfy UF and EP with respect to acl have been of keen interest to model theorists for many years, but the focus has often been on the stable or stable-like setting. Seemingly overlooked until now is that EP implies UF for extensions of DLO (1.17). Hence, by Theorem A,

Corollary. If $T \vDash \mathrm{DC}+\mathrm{EP}$ and extends the theory of densely ordered groups, then $T \vDash \mathrm{UF}$ and every model of $T$ has o-minimal open core.

The converse fails, as we shall see in Section 5 . Indeed, by general model-theoretic reasoning, every extension of the theory of ordered rings having EP satisfies a rather strong condition (1.18) that fails to hold in many interesting cases.

In Section 4, we consider some general results relating $\mathfrak{R}$ and $\mathfrak{R}^{\circ}$. Fundamentally, if $\mathfrak{R}^{\circ}$ is o-minimal, then Boolean combinations of interiors and closures of sets definable in $\mathfrak{R}$ are well behaved. This leads to the notion of quasidecomposition ([26, §5] and [28, pg. 203]), a weak version of cell decomposition. As cell decomposition is arguably the most important basic property of o-minimal structures, one might expect the same to be true of this weaker notion for structures having o-minimal open core. Unfortunately, its utility can be hampered by the possibility of failure of uniformity in parameters, as witnessed by the case that $\mathfrak{R}$ defines a unary function whose graph is somewhere dense. As it turns out (4.16), this is the only obstruction to uniformity in parameters, thus motivating the following.

Definition. We say that $\mathfrak{R}$ has no dense graphs - for short, $\mathfrak{R}$ has NDG or $\mathfrak{R} \vDash$ NDG - if the graph of every definable function is nowhere dense. For a theory $T$, we write $T \vDash$ NDG if every model of $T$ has NDG.

Again, NDG is a first-order schema, and thus is elementary. If every set definable in $\mathfrak{R}$ either has interior or is nowhere dense, then $\mathfrak{R} \vDash$ NDG. In particular, every 
o-minimal structure has NDG. If $\mathfrak{R} \vDash \mathrm{NDG}$ and $\mathfrak{R}^{\circ}$ is o-minimal, then $\mathfrak{R} \vDash \mathrm{EP}$ and $\mathrm{Th}(\mathfrak{R})$ has a complete o-minimal open core (4.15). By 1.17, we also have $\mathfrak{R} \vDash \mathrm{UF}$. Hence, by Theorem A, we have an appealing characterization:

Theorem B. If $T \vDash N D G$ and extends the theory of densely ordered groups, then $T \vDash \mathrm{DC}+\mathrm{UF}$ if and only if $T \vDash \mathrm{DC}+\mathrm{EP}$ if and only if every model of $T$ has o-minimal open core.

Remark. There are many examples of densely ordered structures, even expansions of the real field, that satisfy NDG but whose open core is not o-minimal. We discuss some of them in Section 7.

We have finished describing the main general results of this paper. After proving them, we then focus on several model-theoretic issues related to having o-minimal open core through the prism of some examples and counterexamples. In 28, two natural classes, dense pairs and expansions by generic sets, are given of expansions of the real field that are not o-minimal yet have o-minimal open core; the latter satisfy NDG, while the former do not. In Sections 5 and 6 , we show that these examples extend to our more general setting and we investigate them in more detail, with particular attention paid to EP, definable Skolem functions, elimination of imaginaries, atomic models, and NIP (the "non-independence property"). During the preparation of this paper, some other interesting examples related to dense pairs came to light via Belegradek and Zilber [1,41] and Berenstein, Ealy, and Günaydın [2] we discuss this briefly at the end of Section 5

Having shown that Theorems A and B are nonvacuous, a natural issue is the extent of their optimality. We address this in Section 7 , where we show that the group structure is necessary, even for $R=\mathbb{R}$ and $\mathfrak{R} \vDash$ NDG.

Here is an outline of the rest of this paper. Section 1 is devoted to preliminaries, including global conventions and notation, and some basic topology and model theory. In Section 2] we assume that $\mathfrak{R} \vDash D C$ and develop a number of results, many of which are crucial for the proof of Theorem A, which concludes in Section 3 , Basic properties of structures having o-minimal open core are laid out in Section 4 including specialized results connected with NDG. Some examples and counterexamples are presented in Sections 5 through 7 . We conclude in Section 8 with some open issues.

\section{Preliminaries}

In this section, we declare some global notation and conventions and collect some basic facts to be used later. These fall roughly into two categories, topological and model-theoretic, but as we shall be working over ordered structures, and thus with a definable topology, there is a fair amount of overlap. For the most part, proofs are left as exercises.

The variables $j, k, m, n$ range over $\mathbb{N}$, the nonnegative integers. Given a set $A$, its cardinality is denoted by card $A$. The $n$-th Cartesian power of $A$ is denoted by $A^{n}$, with $A^{0}=\{\emptyset\}$. Where convenient, we identify $A^{m} \times A^{n} \cong A^{m+n}$. Given $C \subseteq A^{m+n}$ and $x \in A^{m}$, we let $C_{x}$ denote the fiber of $C$ over $x$, that is, $C_{x}=$ $\left\{y \in A^{n}:(x, y) \in C\right\}$. Given a collection $\mathcal{A}$ of subsets of $A^{m+n}$ and $x \in A^{m}$, we let $\mathcal{A}_{x}$ denote the collection of fibers $\left\{A_{x}: A \in \mathcal{A}\right\}$. Given a set $B$, we identify a 
function $f: A^{0} \rightarrow B$ with the constant $f(\emptyset) \in B$. Given a function $f: A \rightarrow B$ and $S \subseteq A$, we let $f\lceil S$ denote the restriction of $f$ to $S$.

Generally, $T$ indicates a first-order theory in a language $L$. Whenever convenient, we assume without mention that $L$ contains at least one constant symbol. A first-order structure on a set $M$ is indicated by the corresponding $\mathfrak{M}$, and vice versa. Given $S \subseteq M$, "S-definable (in $\mathfrak{M}$ )" means "definable (in $\mathfrak{M}$ ) with parameters from $S$ ". If no ambient space $M^{n}$ is specified, then " $S$-definable set" means " $S$-definable subset of some $M^{n}$ ", while " $S$-definable function (or map)" means " $S$-definable partial function (or map) from some $A \subseteq M^{m}$ into some $M^{n}$ ". Mention of $S$ is frequently suppressed. A unary set (with respect to $M$ ) is a subset of $M$, and a unary function is one whose graph is contained in $M^{2}$. A point $a \in M^{n}$ is $S$-definable if $a \in \operatorname{dcl}(S)^{n}$, that is, if $\left\{a_{1}, \ldots, a_{n}\right\}$ is $S$-definable. Given structures $\mathfrak{M}_{1}$ and $\mathfrak{M}_{2}$ with common underlying set $M$, we write $\mathfrak{M}_{1}={ }_{\text {df }} \mathfrak{M}_{2}$ if they are interdefinable. QE abbreviates "quantifier elimination". A structure $\mathfrak{M}$ admits $\mathrm{QE}$ if $\operatorname{Th}(\mathfrak{M})$ admits $\mathrm{QE}$.

Recall that $\mathfrak{R}$ denotes a fixed but arbitrary expansion of a dense linear order $(R,<)$ without endpoints. We adjoin formally the endpoints $-\infty$ and $+\infty$ to $R$ in the usual fashion. For our purposes, interval always means nondegenerate interval, that is, an infinite convex $I \subseteq R$ such that both $\inf I$ and $\sup I$ exist in $R \cup\{ \pm \infty\}$. The usual notation is employed for the various kinds of intervals. We sometimes write $R^{>0}$ or $(0, \infty)$ instead of $(0,+\infty)$. Each Cartesian power $R^{n}$ is equipped with the product topology induced by the interval topology on $R$. A box in $R^{n}$ is an $n$-fold product of open intervals, and a closed box is a product of closed intervals. A set has interior if it contains a box; otherwise, we say it has no interior. Limits of functions are always taken with respect to the domain of the function, and similarly with limits superior and inferior. Cells and decompositions relative to $\mathfrak{R}$ are defined as in the o-minimal setting ([10, Chapter 3]).

We shall have occasion to regard $R^{n}$ as lexicographically ordered. When doing so, in order to avoid confusion with $R^{n}$ in the product topology, we add the prefix "lex" to notions involving the order; for example, lexmin $A$ denotes the lexicographic minimum of $A \subseteq R^{n}$ if it exists.

Given $A \subseteq R^{n}$, we denote its interior by $\operatorname{int}(A)$, closure by $\operatorname{cl}(A)$, boundary by $\operatorname{bd}(A)(=\operatorname{cl}(A) \backslash \operatorname{int}(A))$, and frontier by $\operatorname{fr}(A)(=\operatorname{cl}(A) \backslash A)$. If $S \subseteq R$ and $A$ is $S$-definable, then so are each of these sets. We say that $A$ is: constructible if it is a (finite) Boolean combination of open sets; discrete if all of its points are isolated; locally closed if it is open in its closure; nowhere dense if its closure has no interior; somewhere dense if its closure has interior; dense in $C \subseteq R^{n}$ if $\operatorname{cl}(C \cap A)=\operatorname{cl}(C)$; and co-dense in $C$ if $\operatorname{cl}(C \backslash A)=\operatorname{cl}(C)$. We say that $A$ has a locally closed point if there is a box $B \subseteq R^{n}$ such that $\emptyset \neq A \cap B=\operatorname{cl}(A) \cap B$.

1.1. Here is a list of some basic facts from general topology that we shall use.

(1) A set is locally closed if and only if its frontier is closed.

(2) A set is constructible if and only if it is a finite union of locally closed sets. (Consider disjunctive normal form.)

(3) Constructible sets either have interior or are nowhere dense.

(4) Every nonempty constructible set has a locally closed point. (See Dougherty and Miller [5] for a proof.) 
(5) If $f_{1}, \ldots, f_{k}: A \rightarrow R^{n}$ are continuous and $A \subseteq R^{m}$ is locally closed, then the union of their graphs is locally closed. (Show that

$$
\left.\left(A \times R^{n}\right) \cap \bigcup_{i=1}^{k} \operatorname{cl}\left(\operatorname{graph}\left(f_{i}\right)\right) \subseteq \bigcup_{i=1}^{k} \operatorname{graph}\left(f_{i}\right) .\right)
$$

If moreover $B \subseteq R^{n}$ is locally closed, then each $f_{i}^{-1}(B)$ is locally closed. It follows that fibers of locally closed sets are locally closed (though this is easy to check directly).

(6) The previous item holds with "constructible" in place of "locally closed".

(7) There is some useful uniformity associated with (6). Let $A \subseteq R^{m}$ be constructible; say, $A$ is a union of $j$ locally closed sets. Let $f: A \rightarrow R^{n}$ be continuous. Then $f^{-1}(B)$ is a union of $j k$ locally closed sets for every $B \subseteq R^{n}$ that is a union of $k$ locally closed sets. In particular, if $C \subseteq R^{m+n}$ is constructible, then there exists $N \in \mathbb{N}$ such that each fiber $C_{x}$ is a union of $N$ locally closed sets.

(8) Cells and discrete sets are locally closed.

Remarks. (i) Neither complements nor unions of locally closed sets need be locally closed: Both $\left\{(x, y) \in \mathbb{R}^{2}: y \neq 0\right\}$ and $\{(0,0)\}$ are locally closed in $\mathbb{R}^{2}$, their union is not, and the complement of the union is. (ii) Coordinate projections of constructible sets need not be constructible. To illustrate, let $\left(q_{n}\right)_{n \in \mathbb{N}}$ be an enumeration of $\mathbb{Q}$. Regard $A:=\left\{\left(q_{n}, n\right): n \in \mathbb{N}\right\}$ as a subset of $\mathbb{R}^{2}$. Then $A$ is closed and discrete, but its projection onto the first coordinate is dense and co-dense. By regarding $A \subseteq \mathbb{Q}^{2}$, this also shows that a function can have a constructible graph, yet be nowhere continuous.

As fibers of constructible sets are constructible (1.1(6) ),

1.2. If every $\emptyset$-definable set is constructible, then every definable set is constructible.

In combination with 1.1(3),

1.3. If every $\emptyset$-definable set is constructible, then $\mathfrak{R} \vDash N D G$.

1.4. We shall need stronger versions of some of the above facts. By [5], given $S \subseteq R$, every constructible $S$-definable set is a Boolean combination of open $S$-definable sets, hence also a finite union of locally closed $S$-definable sets. Moreover, given $N \in \mathbb{N}$ and $S$-definable $A \subseteq R^{m+n}$, the set

$$
\left\{x \in R^{m}: A_{x} \text { is a union of } N \text { locally closed sets }\right\}
$$

is $S$-definable.

1.5. For $S \subseteq R$, the following are equivalent.

(1) Every open $S$-definable unary set is a finite union of intervals.

(2) Every closed $S$-definable unary set is a finite union of points and intervals.

(3) Every locally closed S-definable unary set is a finite union of points and intervals.

(4) Every constructible S-definable unary set is a finite union of points and intervals.

1.6. $\mathfrak{R} \vDash \mathrm{DC}$ if and only if every open definable unary set is a disjoint union of open intervals. 
1.7. We use the following notation if $\mathfrak{R}$ expands an ordered abelian group. As usual, the identity is denoted by 0 and the inverse operation by - . For $t \in R,|t|$ denotes the absolute value of $t$. For $x \in R^{n}$, put $|x|=\sup \left\{\left|x_{1}\right|, \ldots,\left|x_{n}\right|\right\}$. For $x \in R^{n}$ and $\epsilon>0$, let $B(x, \epsilon)$ denote the box $\left\{y \in R^{n}:|x-y|<\epsilon\right\}$.

1.8. Continuity properties of definable functions play an important role in the sequel. We use the following notation. Given $A \subseteq R^{m}$ and $f: A \rightarrow R^{n}$, let $\mathcal{D}(f)$ denote the set of all $x \in A$ such that $f$ is discontinuous at $x$. Observe that $\mathcal{D}(f)$ is definable in $(R,<, f)$. If $\mathfrak{R}$ expands an ordered abelian group, then for each $s>0$ we put

$$
\mathcal{D}(f, s)=\{x \in A: \forall t>0, f(A \cap B(x, t)) \nsubseteq \subseteq B(f(x), s)\} .
$$

Compare with the usual notion from real analysis: If $R=\mathbb{R}$, then $x \in \mathcal{D}(f, s)$ if and only if the oscillation of $f$ at $x$ is at least $s$. Each $\mathcal{D}(f, s)$ is closed in $A, \mathcal{D}(f)=$ $\bigcup_{s>0} \mathcal{D}(f, s)$, and $(\mathcal{D}(f, s))_{s>0}$ is a decreasing family definable in $(R,<,+, f)$.

$\mathrm{D}_{\Sigma}$ families and sets. The proof of Theorem A relies on a detailed analysis of certain definable sets that are intended to play a role similar to that of the usual $\mathrm{F}_{\sigma}$ sets (that is, countable unions of closed sets) in topology. Recall that every $\mathrm{F}_{\sigma}$ subset of $\mathbb{R}^{n}$ can be expressed as the union of an increasing family of compact sets.

Definition. Let $\mathrm{D}_{\Sigma}(n)$ denote the collection of all indexed families $\left(X_{r, s}\right)_{r, s>0}$ such that $\left\{(r, s, x): r, s>0, x \in X_{r, s}\right\}$ is a definable subset of $R^{>0} \times R^{>0} \times R^{n}$, each $X_{r, s}$ is closed and bounded, and for all $r \leq r^{\prime}$ and $s \geq s^{\prime}$, we have $X_{r, s} \subseteq X_{r^{\prime}, s^{\prime}}$. Observe that if $X:=\left\{(r, s, x): r, s>0, x \in X_{r, s}\right\}$, then $X_{r, s}$ is equal to the fiber $X_{(r, s)}$. We say that a set $A \subseteq R^{n}$ is $\mathrm{D}_{\Sigma}(n)$ if it is equal to the union $\bigcup_{r, s>0} X_{r, s}$ of a $\mathrm{D}_{\Sigma}(n)$ family $\left(X_{r, s}\right)_{r, s>0}$. We suppress the index " $r, s>0$ " whenever reasonable, and typically say " $A \in \mathrm{D}_{\Sigma}(n)$ " or even just " $A$ is $\mathrm{D}_{\Sigma}$ ". Every $\mathrm{D}_{\Sigma}$ set is definable.

$N . B$. In [28], the definition of $\mathrm{D}_{\Sigma}$ uses only the parameter $r>0$. The additional parameter $s$ is necessary here because we shall not require that $\mathfrak{R}$ have a pole. We can eliminate $s$ if there is a definable order-reversing bijection $g: R^{>0} \rightarrow R^{>0}$, for then $\bigcup_{r, s>0} X_{r, s}=\bigcup_{r>0} X_{r, g(r)}$.

If $R=\mathbb{R}$, then every $\mathrm{D}_{\Sigma}$ set is $\mathrm{F}_{\sigma}$, for if $\left(X_{r, s}\right)$ is $\mathrm{D}_{\Sigma}$, then $\bigcup X_{r, s}=\bigcup_{k>0} X_{k, 1 / k}$. Hence, by the Baire Category Theorem, $\bigcup X_{r, s}$ has interior if and only if some $X_{r, s}$ has interior. This result is extremely useful in real topology and analysis; developing an analogue is crucial in the proof of Theorem A. Toward this end, we list in the next two results a number of basic properties of $\mathrm{D}_{\Sigma}$ sets.

1.9. (1) Fibers, finite unions, and finite intersections of $\mathrm{D}_{\Sigma}$ sets are $\mathrm{D}_{\Sigma}$.

(2) If $\left(X_{r, s}\right)$ is $\mathrm{D}_{\Sigma}$ and $a, b \in R^{>0}$, then $\bigcup_{z \in Z} X_{z} \subseteq X_{b, a}$ for every $Z \subseteq$ $[a, b] \times[a, b]$. In particular, for each finite $Z \subseteq R^{>0} \times R^{>0}$ there exist $r, s \in R^{>0}$ such that $\bigcup_{z \in Z} X_{z} \subseteq X_{r, s}$.

(3) If $\left(X_{r, s}\right)$ is $\mathrm{D}_{\Sigma}$ and $\mathfrak{R} \vDash \mathrm{UF}$, then $\bigcup X_{r, s}$ is finite if and only if each $X_{r, s}$ is finite.

Proof. The first two items are immediate from the definition, while the third follows from the second.

Complements of $\mathrm{D}_{\Sigma}$ sets need not be $\mathrm{D}_{\Sigma}$, but counterexamples tend to be rather contrived. To illustrate, let $\left(q_{n}\right)_{n \in \mathbb{N}}$ be an enumeration of $\mathbb{Q}$, and put

$$
X=\left\{\left(r, s, q_{n}\right): n \in \mathbb{N}, r>n, s>0\right\} \subseteq \mathbb{R}^{3} .
$$


Then $\mathbb{Q}=\bigcup X_{r, s}$, so $\mathbb{Q}$ is $\mathrm{D}_{\Sigma}$ in $(\mathbb{R},<, X)$. But $\mathbb{R} \backslash \mathbb{Q}$ is not $\mathrm{F}_{\sigma}$, hence not $\mathrm{D}_{\Sigma}$ in any expansion of $\mathbb{R}$. This example also suggests the possibility of even worse behavior: In $\left(\mathbb{Q},<, X \cap \mathbb{Q}^{3}\right)$, we obtain the open set $\mathbb{Q}$ as the union of a $\mathrm{D}_{\Sigma}$ family of finite sets.

\subsection{Suppose that $\mathfrak{R}$ expands an ordered abelian group.}

(1) If $f: A \rightarrow R^{n}$ is definable and $A \subseteq R^{m}$ is closed, then $\mathcal{D}(f)$ is $\mathrm{D}_{\Sigma}$.

(2) Every constructible definable set is $\mathrm{D}_{\Sigma}$.

(3) If $A \in \mathrm{D}_{\Sigma}(m)$ and $B \subseteq R^{m}$ is constructible and definable, then $A \backslash B \in$ $\mathrm{D}_{\Sigma}(m)$.

Proof. For (11), recall 1.8 and observe that $\mathcal{D}(f)=\bigcup\left([-r, r]^{m} \cap \mathcal{D}(f, s)\right)$. As constructible sets are closed under taking complements, (33) is immediate from (2) and1.9(1). For (2), it suffices by 1.1(11), 1.4, and 1.9(1) to show that if $A$ is definable and $\operatorname{fr}(A)$ is closed, then $A$ is $\mathrm{D}_{\Sigma}$. Let $X$ be the set of all $(r, s, x) \subseteq R^{>0} \times R^{>0} \times A$ such that $|x| \leq r$ and $B(x, s) \cap \operatorname{fr}(A)=\emptyset$. Then $\left(X_{r, s}\right)$ is $\mathrm{D}_{\Sigma}$ and, as $\operatorname{fr}(A)$ is closed, $A=\bigcup X_{r, s}$.

The proof of Theorem A requires only basic topology and first-order definability theory and is independent of the remainder of this section. Hence, the reader may wish to skip now to the next section, returning here later as needed.

1.11. A set $A \subseteq R^{m+n}$, regarded as the parameterized family $\left(A_{x}\right)_{x \in R^{m}}$, has the independence property, or is independent, if for each $k \in \mathbb{N}$ there is a $k$-element $Y \subseteq R^{n}$ such that every subset of $Y$ is of the form $A_{x} \cap Y$ for some $x \in R^{m}$. There are several equivalent formulations, perhaps the most striking being that $A$ does not have the independence property if and only if there exist $d, N \in \mathbb{N}$ such that $\operatorname{card}\left\{A_{x} \cap Y: x \in R^{m}\right\} \leq(\operatorname{card} Y)^{d}$ for all $Y \subseteq R^{n}$ such that $N \leq \operatorname{card} Y<\aleph_{0}$. We write $\mathfrak{R} \vDash$ NIP if no definable set has the independence property, and $T \vDash$ NIP if $\mathfrak{M} \vDash$ NIP for every $\mathfrak{M} \vDash T$. Evidently, $\mathfrak{R} \vDash$ NIP if and only if no $\emptyset$-definable set has the independence property, so NIP is elementary. The notion, introduced by Shelah in the 1970s, has been of keen interest in model theory, but also has connections to probability theory via reinterpretations in terms of Vapnik-Chervonenkis classes. For basic information, see [10, Chapter 5] and Laskowski [20].

1.12. We now recall some crucial basic facts about o-minimality from [19, 35, that will be used often in this paper, either directly or for motivation. Every o-minimal structure satisfies all of DC, UF, EP, NIP, and NDG. (Actually, NDG is not addressed in those papers, but it follows immediately from cell decomposition.) Ominimality is elementary. (This requires effort, in contrast to the elementarity of DC, UF, NDG, and NIP, all of which are easily expressed as first-order schema.) Hence, a complete theory is o-minimal if it has an o-minimal model. (A theory is o-minimal if all of its models are o-minimal.) Every complete o-minimal theory has a unique up-to-isomorphism prime model, and this prime model is also atomic.

Convention. From now on, expansions of ordered groups are regarded as equipped with a distinguished positive element, typically denoted by 1 . All results stated so far, including those in the introduction, are independent of this convention. We let DOG denote the theory of densely ordered groups with a distinguished positive element. 
1.13. Suppose that $\mathfrak{R}$ expands a densely ordered group and is o-minimal.

(1) "Definable Choice" [10, Chapter 6]: Let $A \subseteq R^{m+n}$ be $\emptyset$-definable and $\pi A$ be the projection of $A$ onto the first $m$ variables. Then there is a $\emptyset$-definable map $f: \pi A \rightarrow R^{n}$ whose graph is contained in $A$. Moreover, $f(x)=f(y)$ for all $x, y \in \pi A$ such that $A_{x}=A_{y}$. It follows that $\operatorname{Th}(\Re)$ has definable Skolem functions and eliminates imaginaries; indeed, every definable equivalence relation has a definable set of representatives (over the same set of parameters).

(2) Extractable from Peterzil and Starchenko [32, §7]: If $\mathfrak{R}$ has a pole, then there exist unique $\emptyset$-definable binary operations $\oplus, \odot$ such that the structure $(R,<, \oplus, \odot, 0,1)$ is an ordered field.

(3) Edmundo [14]: If $\mathfrak{R}$ has no poles, then $\mathfrak{R}$ is interdefinable with an expansion of an ordered division ring by bounded sets. A detailed structure theorem shows that the definable sets are rather simple.

(4) Laskowski and Steinhorn 21: If $\operatorname{Th}(\mathfrak{R})$ has an Archimedean model, then it has a model over $\mathbb{R}$ that is unique up to isomorphism.

In light of (2), (3), and the Trichotomy Theorem [32, we might be justified in focussing this paper on expansions of ordered fields, see also Miller and Starchenko [29], but we work in greater generality whenever reasonable. Of course, (4) shows that many of the results of this paper are redundant over [28] in the Archimedean setting, yet even the reader interested only in expansions of the real field will find herein new results and techniques.

The next result is due essentially to van den Dries 1

1.14. (1) If every $\emptyset$-definable unary set is a finite union of points and intervals, and every $\emptyset$-definable open interval intersects $\operatorname{dcl}(\emptyset)$, then $\Re$ is o-minimal.

(2) If $\mathfrak{R}$ expands a divisible ordered group and every $\emptyset$-definable unary set is a finite union of points and intervals, then $\mathfrak{R}$ is o-minimal.

(Compare (1) with [28, Proposition 1].)

Proof. (1). By induction on the dimension of the ambient space, every nonempty $\emptyset$-definable set in $R^{n}$ intersects $\operatorname{dcl}(\emptyset)^{n}$. By Vaught's Test, $\operatorname{dcl}(\emptyset)$ is the underlying set of an elementary substructure $\mathfrak{M}$ of $\mathfrak{R}$. Definability and $\emptyset$-definability are the same in $\mathfrak{M}$, so $\mathfrak{M}$ is o-minimal. As o-minimality is elementary, $\mathfrak{R}$ is o-minimal.

(2). Observe that if $a, b \in R$, then all of $1, a+1, b-1$, and $(a+b) / 2$ are $\{a, b\}$-definable. Hence, every $\emptyset$-definable open interval intersects $\operatorname{dcl}(\emptyset)$. Now apply (1).

Remark. The assumption in (1) that every $\emptyset$-definable open interval intersects $\operatorname{dcl}(\emptyset)$ is necessary. To illustrate, the expansion of $(\mathbb{R},<)$ by the Vitali relation $\{(x, y) \in$ $\left.\mathbb{R}^{2}: x-y \in \mathbb{Q}\right\}$ admits $\mathrm{QE}$ (an exercise), so $\emptyset$ and $\mathbb{R}$ are the only $\emptyset$-definable unary sets.

1.15. We shall have peripheral reasons to mention two generalizations of o-minimality. The structure $\mathfrak{R}$ is weakly o-minimal if every definable unary set is a finite union of convex sets, and is locally o-minimal if for every definable unary $A$ and $x \in R$ there is an open interval $I$ about $x$ such that $A \cap I$ is a finite union of points

\footnotetext{
${ }^{1}$ Communicated to Miller by van den Dries in personal correspondence. Neither published nor submitted elsewhere as yet.
} 
and intervals, equivalently, if there is an open interval $I$ about $x$ such that $A \cap I \backslash\{x\}$ is one of the following sets: $\emptyset, I \backslash\{x\},(x, \infty) \cap I$, or $(-\infty, x) \cap I$. (It follows that local o-minimality is elementary.) A theory is weakly, or locally, o-minimal if the same is true of all of its models. Evidently, o-minimality $\Rightarrow$ weak o-minimality $\Rightarrow$ local o-minimality. It is easy to see that none of the converses holds. (Consider the structures $(\mathbb{Q},<, \mathbb{Z})$ and $(\{0,1\} \times \mathbb{Q},<,\{0\} \times \mathbb{Q})$. More substantial examples are given in Section 7 ) If $\mathfrak{R}$ is weakly o-minimal, then every unary definable set either has interior or is finite. If $\mathfrak{R}$ is locally o-minimal, then every unary definable set either has interior or is closed and discrete. Again, the converses fail, but do hold if $\Re \vDash D C(2.11$, 2.12). For more information on weak o-minimality, see [24] and its bibliography. As of this writing, local o-minimality is only just beginning to be documented in the literature (C. Toffalori and K. Vozoris [40]); we shall prove only a few results that arise as byproducts of our investigation of open cores.

We close this section with some miscellaneous model-theoretic results.

1.16. If $\mathfrak{R}$ is $\aleph_{0}$-saturated and $\mathfrak{R} \not \nvdash \mathrm{UF}$, then $\mathfrak{R}$ defines a "nonstandard finite set", that is, an infinite $Y \subseteq R$ such that for every $X \subseteq Y$ definable in $\mathfrak{R}$ and $x \in X$, either $x=\min X$ or $x$ has a predecessor in $X$, and either $x=\max X$ or $x$ has a successor in $X$. Hence, if no $\mathfrak{M} \equiv \mathfrak{R}$ defines an infinite discrete unary set, then $\mathfrak{R} \vDash \mathrm{UF}$. As an easy consequence, every weakly o-minimal theory has UF.

1.17. If $\mathrm{DLO} \subseteq T \vDash \mathrm{EP}$, then $T \vDash \mathrm{UF}$.

Proof. Suppose to the contrary that $T \not \models$ UF. By 1.16, there is a $(\operatorname{card} T)^{+}$-saturated model of $T$ that defines an infinite discrete unary set $A$ using parameters from a set $S$. By saturation, there exists $a \in A \backslash \operatorname{dcl}(S)$. As $A$ is discrete, there is an interval $I$ such that $I \cap A=\{a\}$. Again by saturation, there exists $b \in I \backslash \operatorname{dcl}(S \cup\{a\})$ such that $a<b$. Now, $a=\max (A \cap(-\infty, b))$, so $a \in \operatorname{dcl}(S \cup\{b\}) \backslash \operatorname{dcl}(S)$, but then $b \in \operatorname{dcl}(S \cup\{a\})$ by EP, a contradiction.

1.18. Suppose that $T$ extends the theory of ordered rings.

(1) If $T \vDash \mathrm{EP}$, then $T$ defines a function $F$ of four variables in the language of rings such that $F\left(A^{4}\right)=M$ for every $\mathfrak{M} \vDash T$ and definable infinite $A \subseteq M$.

(2) If $T \vDash \mathrm{EP}$ and $\mathfrak{M} \vDash T$, then $\operatorname{card} A=\operatorname{card} M$ for every infinite unary set A definable in $\mathfrak{M}$.

(3) No extension of the theory of proper elementary pairs of models of $T$ has $\mathrm{EP}$.

Proof. (1). Suppose that $T \vDash$ EP. It suffices to work in a fixed $(\operatorname{card} T)^{+}$-saturated model $\mathfrak{M}$ of $T$. As ordered rings have no zero divisors, given $x \in M^{4}$ with $x_{1} \neq x_{3}$, there is at most one $t \in M$ such that $t x_{1}+x_{2}=t x_{3}+x_{4}$. Define $F$ by

$$
x \mapsto \begin{cases}t, & \text { if } x_{1} \neq x_{3} \text { and } t x_{1}+x_{2}=t x_{3}+x_{4}, \\ 0, & \text { otherwise. }\end{cases}
$$

Let $A \subseteq M$ be infinite and definable. We show that $F\left(A^{4}\right)=M$. Suppose not. Let $S$ be the parameters used in defining $A$. By saturation, there exist $a, b \in A$ and $c \in M \backslash F\left(A^{4}\right)$ such that the dcl-rank of $\{a, b, c\}$ over $S$ is 3 . As $c \notin F\left(A^{4}\right)$, the function $\left(x_{1}, x_{2}\right) \mapsto c x_{1}+x_{2}: A^{2} \rightarrow M$ is injective, so the point $(a, b)$ is the unique solution in $A^{2}$ to the equation $c x_{1}+x_{2}=c a+b$. Thus, $\{a, b\}$ is dependent over $S \cup\{c, c a+b\}$. But then the dcl-rank of $\{a, b, c\}$ over $S$ is at most 2, a contradiction.

Items (2) and (3) are immediate from (1). 
Remarks. (i) We shall see in Section 5 that the converse of 1.17 fails, and 1.18 fails if "ring" is relaxed to "vector space". (ii) The only use of the underlying order in the proof of 1.18 is to conclude that the ring has no zero divisors. Hence, the conclusion holds for any theory extending that of rings without zero divisors. Moreover, it suffices to assume that EP is taken with respect to acl instead of dcl. (iii) It appears to us that 1.17 and 1.18 have hitherto gone unnoticed.

1.19. For sets $X, Y$ and $A \subseteq X$, we say that a function $f: A \rightarrow Y$ is given piecewise by a collection $\mathcal{F}$ of functions $X \rightarrow Y$ if there is a finite $\mathcal{G} \subseteq \mathcal{F}$ such that $\operatorname{graph}(f) \subseteq \bigcup_{g \in \mathcal{G}} \operatorname{graph}(g)$.

1.20. Given theories $T$ and $T^{\prime}$, we say that dcl in $T^{\prime}$ is the same as in $T$, or $\mathrm{dcl}_{T}=\mathrm{dcl}_{T^{\prime}}$ for short, if $\operatorname{dcl}_{\mathfrak{M}}=\operatorname{dcl}_{\mathfrak{M}^{\prime}}$ for all $\mathfrak{M} \vDash T$ and $\mathfrak{M}^{\prime} \vDash T^{\prime}$ having common underlying set $M$. Suppose that $\mathrm{dcl}_{T}=\mathrm{dcl}_{T^{\prime}}$. A routine compactness argument yields that for every $\mathfrak{M} \vDash T$ and $\mathfrak{M}^{\prime} \vDash T^{\prime}$ having common underlying set $M$, and for every $S \subseteq M$, every function $S$-definable in $\mathfrak{M}$ is given piecewise by functions $S$-definable in $\mathfrak{M}^{\prime}$, and every function $S$-definable in $\mathfrak{M}^{\prime}$ is given piecewise by functions $S$-definable in $\mathfrak{M}$. (The converse holds as well, of course.) Hence, if moreover $T$ and $T^{\prime}$ extend DLO, then $T \vDash$ NDG if and only if $T^{\prime} \vDash$ NDG. (Finite unions of nowhere dense sets are nowhere dense.) In particular, if $T$ is o-minimal and $T^{\prime} \supseteq \mathrm{DLO}$, then $T^{\prime} \vDash \mathrm{EP}+\mathrm{UF}+\mathrm{NDG}$ (recall 1.12 and 1.17). On the other hand, we shall see that for every complete o-minimal $T$ extending the theory of ordered fields there are complete theories $T_{1}, T_{2}, T_{3} \supseteq T$, all satisfying UF, such that: (i) $T_{1} \vDash \mathrm{DC}$ and $T_{1} \not \models \mathrm{EP}$ (Section 5); (ii) $T_{2} \vDash \mathrm{DC}$, $\mathrm{dcl}_{T_{2}}=\mathrm{dcl}_{T}$, and $T_{2}$ is not o-minimal (Section 6); and (iii) $\mathrm{dcl}_{T_{3}}=\operatorname{dcl}_{T}$ and $T_{3} \not \models \mathrm{DC}$ (Section 7, convex pairs).

\section{Definable completeness}

Throughout this section, we assume that $\mathfrak{R} \vDash$ DC, equivalently (1.6), that every open definable unary set is a disjoint union of open intervals. This enables the development of a substantial amount of elementary point-set topology as if we were working over the real line. (Precursors in the o-minimal setting of some of these results are found in various places, in particular, $[10,12,33,35$.$) Indeed, some$ results need so little modification that we simply mark them with an $(\mathbb{R})$ and omit the proofs. We first concentrate on results needed for the proof of Theorem A, then follow up with some other miscellaneous facts to be used in later sections.

We say that a subset of $R^{n}$ is CBD if it closed, bounded and definable. CBD sets play a role here similar to that of compact sets in real topology. We recall some basic facts from [25].

2.1. (1) If $\emptyset \neq A \subseteq R^{m}$ is $C B D$, then lexmin $A$ and lexmax $A$ exist.

(2) If $\emptyset \neq A \subseteq R^{m}$ is $C B D$ and $f: A \rightarrow R^{n}$ is continuous and definable, then $f(A)$ is $C B D$, and there exist $x, y \in A$ such that $f(x)=\operatorname{lexmin} f(A)$ and $f(y)=\operatorname{lexmax} f(A)$.

(3) If $\Re$ expands a densely ordered group, then the group is abelian and divisible.

Remark. See [4] for a partial converse to (2).

As an immediate consequence of (2),

2.2. If $A$ is $\mathrm{D}_{\Sigma}$ and $f: A \rightarrow R^{n}$ is continuous and definable, then $f(A)$ is $\mathrm{D}_{\Sigma}$. In particular, coordinate projections of $\mathrm{D}_{\Sigma}$ sets are $\mathrm{D}_{\Sigma}$. 
2.3. (R) Let $A \subseteq R^{m}$ and $f: A \rightarrow R^{n}$ be definable. Then $f$ is continuous if and only if its graph is closed in $A \times R^{n}$ and for every $x \in A$ there is a bounded box $U \times V$ about $(x, f(x))$ such that $f(A \cap U) \subseteq V$.

\subsection{The following are equivalent.}

(1) $\mathfrak{R} \vDash U F$.

(2) For every definable $A \subseteq R^{m+n}$ there exists $N \in \mathbb{N}$ such that for all $x \in R^{m}$, if $A_{x}$ is discrete and closed, then card $A_{x} \leq N$.

(3) For every definable $A \subseteq R^{m+1}$ there exists $N \in \mathbb{N}$ such that for all $x \in R^{m}$, if $A_{x}$ is discrete and closed, then card $A_{x} \leq N$.

Remark. We show in 7.3 below that "closed" is necessary, even in the presence of NDG.

Proof. As finite sets are discrete and closed, $(2) \Rightarrow(3) \Rightarrow(1)$ is clear. We now show that $(1) \Rightarrow(2)$. By UF, it suffices to consider the case $m=0$. Let $A \subseteq R^{n}$ be definable, discrete and closed. We show that $A$ is finite. As $A=\bigcup_{a, b \in R}\left(A \cap[a, b]^{n}\right)$ and subsets of discrete sets are discrete, we reduce again by UF to the case that $A$ is bounded. Suppose to the contrary that $A$ is infinite. Let $B$ be the set of all $(x, y) \in A \times A$ such that $x \geq y$ in the lexicographic order. Observe that $B$ is definable. By 2.1. put $a_{0}=\operatorname{lexmin} A$; then $B_{a_{0}}=\left\{a_{0}\right\}$. Now, $A \backslash\left\{a_{0}\right\}$ is again infinite, discrete, and CBD, so we put $a_{1}=\operatorname{lexmin}\left(A \backslash\left\{a_{0}\right\}\right)$ and we have $B_{a_{1}}=\left\{a_{0}, a_{1}\right\}$. Proceeding inductively, we obtain a sequence $\left\{a_{k}\right\}$ of distinct elements of $A$ such that $B_{a_{k}}=\left\{a_{0}, \ldots, a_{k}\right\}$ for each $k$, violating UF.

2.5. Lemma. Suppose that $\mathfrak{R}$ expands a densely ordered group.

(1) The following are equivalent.

(a) Every discrete definable unary set is finite.

(b) Every constructible definable unary set is a finite union of points and intervals.

(2) The following are equivalent.

(a) $\mathfrak{R} \vDash \mathrm{UF}$.

(b) For every definable $A \subseteq R^{m+n}$ there exists $N \in \mathbb{N}$ such that for all $x \in R^{m}$, if $A_{x}$ is discrete, then card $A_{x} \leq N$.

(c) If $A \subseteq R^{m+1}$ is definable, then there exist $M, N \in \mathbb{N}$ such that for all $x \in R^{m}, \operatorname{int}\left(A_{x}\right)$ is a disjoint union of at most $N$ open intervals, and $\operatorname{cl}\left(A_{x}\right)$ is a disjoint union of at most $M$ points and $N$ closed intervals.

Remark. We show in 7.4 below that DC is necessary, even in the presence of NDG.

Proof. (1). The set

$$
\{x \in U: \exists \epsilon>0,(x-\epsilon, x+\epsilon) \subseteq U \text { \& } x-\epsilon, x+\epsilon \in \operatorname{bd}(U)\}
$$

is definable and discrete. Now apply 1.5 and 1.6 .

(2). By 1.1 and 1.10, every discrete definable set is the union of a $\mathrm{D}_{\Sigma}$ family of discrete CBD sets. The result is now immediate from 1.9.3) and 2.4

2.6. Corollary. Let DLO $\subseteq T \vDash \mathrm{DC}$. Then $T \vDash \mathrm{UF}$ if and only if every closed discrete set definable in any model of $T$ is finite. If moreover $T$ extends the theory of densely ordered groups, then $T \vDash \mathrm{UF}$ if and only if every discrete set definable in any model of $T$ is finite.

Next is a key intermediate result inspired by the proof of [28, Theorem (b)]. 
2.7. If $\Re$ expands a densely ordered group and every $\mathrm{D}_{\Sigma}$ set is constructible, then:

(1) $\mathfrak{R}^{\circ}$ is interdefinable with the expansion of $R$ by the $\mathrm{D}_{\Sigma}$ sets definable in $\mathfrak{R}$.

(2) Every set definable in $\mathfrak{R}^{\circ}$ is constructible.

(3) The following are equivalent.

(a) $\mathfrak{R}^{\circ}$ is o-minimal.

(b) Every open definable unary set is a finite union of intervals.

(c) Every discrete definable unary set is finite.

Proof. By 1.10, every constructible definable set is $\mathrm{D}_{\Sigma}$, so (1) is immediate. For (2), we proceed by induction on formulas. Every set that is quantifier-free definable in $\mathfrak{R}^{\circ}$ is constructible. As constructible sets are closed under taking complements, it now suffices to show that the constructible definable sets are closed under projections. Again, all constructible definable sets are $D_{\Sigma}$. By $2.2 D_{\Sigma}$ sets are closed under projections. As we have assumed that all $\mathrm{D}_{\Sigma}$ sets are constructible, we are done. For (3), combine (2) with 2.5)(11).

By combining Lemma 2.5 and 2.7 the strategy for the proof of Theorem A is revealed: Assuming that $\mathfrak{R} \vDash \mathrm{DC}+\mathrm{UF}$ and expands a densely ordered group, we show that every $\mathrm{D}_{\Sigma}$ set is constructible. We do this in the next section.

It is not quite fair to say that the next result follows just as in real analysis, because one usually proves something stronger straightaway via the combination of separability and the Baire Category Theorem. Nevertheless, the best way to understand the proof is to think of working over $\mathbb{R}$. Recall the notation from 1.8

2.8. Let $\mathfrak{R}$ expand a densely ordered group. Fix $m \in \mathbb{N}$ and let $\pi$ denote projection onto the first $m$ coordinates. Let $s>0$ and $A \subseteq R^{m+n}$ be $C B D$.

(1) If $A \neq \emptyset$, then the map $f(x):=\operatorname{lexmin} A_{x}$ is defined on $\pi A$, and $\mathcal{D}(f, s)$ is nowhere dense.

(2) If the set $\left\{x \in R^{m}: A_{x}\right.$ contains a box of side length $\left.s\right\}$ has interior, then $A$ has interior.

(3) For each coordinate projection $\rho: R^{n} \rightarrow R$, the set

$$
S_{\rho}:=\left\{x \in R^{m}: \rho A_{x} \text { contains a closed interval of length } s\right\}
$$

is $C B D$.

Remark. Item (2) answers a question left open in [25].

Proof. (11). Each fiber $A_{x}$ with $x \in R^{m}$ is CBD. Hence, the map $f$ exists by 2.1 We now show by induction on $n \geq 1$ that $\mathcal{D}(f, s)$ is nowhere dense.

Let $n=1$. As $A$ is CBD, $f$ is bounded, so $f(x) \leq \liminf _{y \rightarrow x} f(y)$ for all $x \in \pi A$. Hence, $f$ is lower semicontinuous, that is, $f^{-1}((c, \infty))$ is open in $\pi A$ for each $c \in R$. Let $B \subseteq R^{m}$ be a box that intersects $\pi A$. Put $M=\sup f(B \cap \pi A)$ and choose $x \in B \cap \pi A$ such that $f(x)>M-s$. By lower semicontinuity, there is a box $B^{\prime}$ about $x$ such that $f\left(B^{\prime} \cap \pi A\right) \subseteq(M-s, M]$. Then $B^{\prime}$ is disjoint from $\mathcal{D}(f, s)$. As $B$ was arbitrary, $\mathcal{D}(f, s)$ is nowhere dense.

Assume the result for a certain $n \geq 1$ and let $A \subseteq R^{m+n+1}$ be CBD. Let $C$ be the projection of $A$ onto the first $m+n$ coordinates. By the $n=1$ case, $\mathcal{D}(g, s)$ is nowhere dense, where $g(x, y)=\min A_{(x, y)}$ for $(x, y) \in C$. By 2.1, $C$ is CBD. Inductively, $\mathcal{D}(h, s)$ is nowhere dense, where $h(x)=\operatorname{lexmin} C_{x}$ for $x \in \pi C(=\pi A)$. Hence, $\mathcal{D}(f, s)$ is nowhere dense. 
(2). We shall only invoke this result for $n=1$; for ease of notation, we do only this case. Let $D$ be the set of all $d>0$ such that, for some box $B \subseteq \pi A$, every $A_{x}$ with $x \in B$ contains an interval of length $d$. Now, $A$ is bounded and definable; hence so is $D$. Moreover, $s \in D$, so $d:=\sup D$ exists in $R$. Hence, $\pi A$ contains a box $B$ such that every $A_{x}$ with $x \in B$ contains a closed interval of length $3 d / 4$, but no interval of length greater than $d$. Define $g: B \rightarrow R$ by

$$
g(x)=\inf \left\{t \in R:[t, t+3 d / 4] \subseteq A_{x}\right\} .
$$

Similar to the $n=1$ case of (1), $g$ is bounded and we have $g(x) \leq \liminf _{y \rightarrow x} g(y)+$ $d / 4$ for all $x \in B$. Put $M=\sup g$ and choose $x \in B$ such that $g(x)>M-d / 4$. Then there is a box $B^{\prime} \subseteq B$ about $x$ such that $g\left(B^{\prime}\right) \subseteq(M-d / 2, M)$. Hence, $B^{\prime} \times(M, M+d / 2) \subseteq A$.

(3). It suffices to show that $S_{\rho}$ is closed. Let $y \in \operatorname{cl}\left(S_{\rho}\right)$. Each $A_{x}$ is CBD, hence also each $\rho A_{x}$ by 2.1. Put $b=\liminf _{x \rightarrow y} \min \left\{t \in R:[t, t+s] \subseteq \rho A_{x}\right\}$. As $A$ is $\mathrm{CBD}$, we have $[b, b+s] \subseteq \rho A_{y}$, that is, $y \in S_{\rho}$.

The proof of Theorem A does not depend on the remainder of this section.

\subsection{Cells of $\mathfrak{R}$ are definably connected.}

(Compare with [10, 3.2.9].)

Proof. It is immediate from DC that intervals are definably connected, so the Intermediate Value Theorem holds for definable functions [25]. The rest is an easy induction.

2.10. ( $\mathbb{R})$ If $A \subseteq R$ is definable, closed, and has neither interior nor isolated points, then it is the union of the sets

$$
\begin{aligned}
& \{x \in A: x \in \operatorname{cl}(A \cap(x, \infty)) \backslash \operatorname{cl}(A \cap(-\infty, x))\}, \\
& \{x \in A: x \in \operatorname{cl}(A \cap(-\infty, x)) \backslash \operatorname{cl}(A \cap(x, \infty))\}, \\
& \{x \in A: x \in \operatorname{cl}(A \cap(-\infty, x)) \cap \operatorname{cl}(A \cap(x, \infty))\},
\end{aligned}
$$

each of which is dense in $A$ (and thus has no locally closed points).

\subsection{The following are equivalent.}

(1) $\mathfrak{R}$ is locally o-minimal.

(2) Every definable unary set either has interior or is closed and discrete.

(3) Every definable unary set is constructible, and is closed if discrete.

(4) Every nonempty definable unary set has a locally closed point, and is closed if discrete.

Proof. We show that $(1) \Rightarrow(2) \Rightarrow(3) \Rightarrow(4) \Rightarrow(2) \Rightarrow(1)$. Let $A \subseteq R$ be definable.

$(1) \Rightarrow(2)$. Suppose that $A$ has no interior. Let $x \in A$. By local o-minimality, there exist $a<x<b$ such that $A \cap(a, b)$ is finite. It follows that $A$ is discrete and has no limit points, and thus is closed as well.

For $(2) \Rightarrow(3)$, put $B=A \backslash \operatorname{int}(A)$. Then $B$ is closed by assumption, so $A$ is constructible. For $(3) \Rightarrow(4)$, apply 1.1(4).

$(4) \Rightarrow(2)$. First, suppose that $A$ is closed. We must show that $A$ is discrete. Let $B$ be the set of isolated points of $A$. We are done if $A=B$, so let $A \neq B$. As $B$ is definable and discrete, it is closed by assumption. Hence, $A \backslash B$ is nonempty, closed, and has no isolated points. By assumption, $A \backslash B$ has a locally closed point. By 2.10, $A \backslash B$ has interior, hence so does $A$. For the general case, $\operatorname{bd}(A)$ is closed 
and has no interior, and thus is closed and discrete. If $A \subseteq \operatorname{bd}(A)$, then $A$ is closed and discrete. If $A \nsubseteq \mathrm{bd}(A)$, then $A$ has interior.

$(2) \Rightarrow(1)$. Let $x \in R$. We must find $a<x<b$ such that $A \cap(a, b)$ is a finite union of points and open intervals. It suffices to consider the case that $x \in \operatorname{bd}(A)$. As $\operatorname{bd}(A)$ has no interior, it is discrete by assumption. Then there exist $a<x<b$ such that $\operatorname{bd}(A) \cap(a, b)=\{x\}$. By DC, we may assume that $a, b \in \operatorname{bd}(A) \cup\{ \pm \infty\}$. Hence, $A \cap(a, b)$ is equal to one of $\{x\},(a, x),(a, x],[x, b),(x, b)$, or $(a, b) \backslash\{x\}$.

\subsection{The following are equivalent.}

(1) $\mathfrak{R}$ is o-minimal.

(2) Every definable unary set either has interior or is finite.

(3) Every definable unary set is constructible, and is finite if discrete.

(4) Every nonempty definable unary set has a locally closed point, and is finite if discrete.

(5) $\operatorname{Th}(\mathfrak{R}) \vDash \mathrm{EP}$ and every definable unary set either has interior or is nowhere dense.

(6) $\mathfrak{R}$ is locally o-minimal and every discrete definable unary set is finite.

(7) $\mathfrak{R} \vDash \mathrm{UF}$ and is locally o-minimal.

(8) $\mathfrak{R}$ is weakly o-minimal.

Proof. The equivalence of the first four conditions is essentially the same as in 2.11. All o-minimal structures satisfy both EP and UF, disposing of $(1) \Rightarrow[(5) \&(7)]$. An argument similar to that of 1.17 yields $(5) \Rightarrow(2)$. By 2.4 , we have $(7) \Rightarrow(6)$. Finally, $(6) \Rightarrow(1)$ follows from 1.6

The case $R=\mathbb{R}$. Suppose now that $\mathfrak{R}$ is an expansion of the real line. Of course, the assumption of DC is superfluous.

2.13. (1) The previous two results hold with " $\mathrm{F}_{\sigma}$ " in place of "constructible", since every nonempty subset of $\mathbb{R}$ that is both $\mathrm{F}_{\sigma}$ and $\mathrm{G}_{\delta}$ has a locally closed point. See [26, 3.1] for more information.

(2) If every infinite definable unary set is uncountable, then $\mathfrak{R}^{\circ}$ is o-minimal [28. Hence, $\mathfrak{R}$ is o-minimal if and only if $\mathfrak{R}={ }_{\mathrm{df}} \mathfrak{R}^{\circ}$ and every infinite definable unary set is uncountable.

(3) If $\mathfrak{R}$ expands the real field and is locally o-minimal, then it is o-minimal. To see this, let $A \subseteq \mathbb{R}$ be definable and have no interior. Then the same is true of $\{1 / a: 0 \neq a \in A\}$, so this set is closed and discrete (2.11). Thus, $A$ is bounded, closed and discrete, hence finite by Bolzano-Weierstrass.

We have an improvement of [28, Corollary (2)], which was established under the additional assumption of $\mathfrak{R}$ having a pole. In essence, UF becomes superfluous in Theorem A.

2.14. Proposition. If $\Re$ expands $(\mathbb{R},<,+)$, then the following are equivalent.

(1) $\mathfrak{R}^{\circ}$ is o-minimal.

(2) Every open definable unary set is a finite union of intervals.

(3) Every discrete definable unary set is finite.

Remark. We show in 7.1 below that the group structure is necessary, even in the presence of NDG. 
Proof. By 2.7, it suffices to assume that every open definable unary set is a finite union of intervals, and then show that every $\mathrm{D}_{\Sigma}$ set is constructible, using our definition of $\mathrm{D}_{\Sigma}$. By examination of the proof of [28, Theorem (b)], it suffices to show that every $A \in \mathrm{D}_{\Sigma}(1)$ is a finite union of points and intervals. As $\operatorname{int}(A)$ is open and definable, it is a finite union of intervals. Hence, it suffices to show that $A \backslash \operatorname{int}(A)$ is finite. By 1.10, $A \backslash \operatorname{int}(A)$ is $\mathrm{D}_{\Sigma}$. Write $A \backslash \operatorname{int}(A)=\bigcup X_{r, s}$ as usual. Each $X_{r, s}$ is closed, definable and has no interior, and thus is finite. Put $Y=\left\{(s, t): t \in \bigcup_{r>0} X_{r, s}\right\}$. Observe that $Y$ is definable, $Y_{s}=\bigcup_{r>0} X_{r, s}$ for each $s>0$, and the family $\left(Y_{s}\right)_{s>0}$ is decreasing. By [28, 2.3], each $Y_{s}$ is finite. By a routine modification of the proof, so is $\bigcup_{s>0} Y_{s}(=A \backslash \operatorname{int}(A))$.

Remark. We take this opportunity to point out that the first sentence immediately following [28, Corollary, p. 194] should be interpreted as saying that the corollary holds with "Ø-definable" in place of "definable" including in the definition of $\mathfrak{R}^{\circ}$. The import of this clarification will be revealed below in Section 4, subsection "Specializing to sets of parameters".

\section{Proof of Theorem A}

Throughout this section, we assume that $\mathfrak{R} \vDash \mathrm{DC}+\mathrm{UF}$ and expands a densely ordered group. We show that $\mathfrak{R}^{\circ}$ is o-minimal and is interdefinable with the expansion of $R$ by the $D_{\Sigma}$ sets of $\Re$. By Lemma 2.5 and 2.7, we need only show that every $\mathrm{D}_{\Sigma}$ set is constructible. Our proof proceeds through a number of steps. First, we have an analog of a key property of $\mathrm{F}_{\sigma}$ subsets of $\mathbb{R}^{n}$, namely, that the union of a countable family $\left\{F_{k}\right\}$ of closed subsets of $\mathbb{R}^{n}$ has interior if and only if some $F_{k}$ has interior.

3.1. Let $A=\bigcup X_{r, s} \in \mathrm{D}_{\Sigma}(n)$. Then either $A$ has no interior or some $X_{r, s}$ has interior. If $n=1$, then either $A$ is finite or some $X_{r, s}$ has interior.

Proof. We proceed by induction on $n \geq 1$.

Let $n=1$. Suppose that no $X_{r, s}$ has interior. Each $X_{r, s}$ is CBD, hence finite by 2.5(2). Then $A$ is finite by UF and 1.9 .

Assume the result for $n \geq 1$ and let $A \in \mathrm{D}_{\Sigma}(n+1)$ have interior. Then $A$ contains a bounded closed box $B \subseteq R^{n+1}$. As $B=\bigcup\left(B \cap X_{r, s}\right)$ and each $B \cap X_{r, s}$ is $\mathrm{CBD}$, we may assume that $A$ is a bounded closed box $C \times I \subseteq R^{n} \times R$. By Lemma 2.5, there exists $N \in \mathbb{N}$ such that for all $(r, s, x) \in R^{2+n}$, the fiber $X_{r, s, x}$ is a disjoint union of finitely many points and at most $N$ closed intervals. (For ease of notation, we write $X_{r, s, x}$ rather than $X_{(r, s, x)}$.) Write $I$ as a union of $2 N+1$ closed intervals $I_{k}$ whose interiors are pairwise disjoint. For each fixed $(r, s, k)$, the set $\left\{x \in R^{n}: I_{k} \subseteq X_{r, s, x}\right\}$ is CBD. Hence, for each fixed $k$, the family

$$
\left(\left\{x \in R^{n}: I_{k} \subseteq X_{r, s, x}\right\}\right)_{r, s>0}
$$

is $\mathrm{D}_{\Sigma}$, so the same is true of the family

$$
\left(\bigcup_{k=1}^{2 N+1}\left\{x \in R^{n}: I_{k} \subseteq X_{r, s, x}\right\}\right)_{r, s>0} .
$$

It suffices now to show that

$$
C=\bigcup_{r, s>0} \bigcup_{k=1}^{2 N+1}\left\{x \in R^{n}: I_{k} \subseteq X_{r, s, x}\right\},
$$


for then, inductively, there exist $a, b>0$ such that

$$
\bigcup_{k=1}^{2 N+1}\left\{x \in R^{n}: I_{k} \subseteq X_{a, b, x}\right\}
$$

has interior, so there exists $k \in\{1, \ldots, 2 N+1\}$ such that $\left\{x \in R^{n}: I_{k} \subseteq X_{a, b, x}\right\}$ has interior. Hence, $X_{a, b}$ has interior.

Fix $x \in C$ and put $Y_{r, s}=X_{r, s, x}$ for $r, s>0$. We must produce $k \in\{1, \ldots, 2 N+$ $1\}$ and $a, b>0$ such that $I_{k} \subseteq Y_{a, b}$. Let $k \in\{1, \ldots, 2 N+1\}$. The family $\left(I_{k} \cap Y_{r, s}\right)$ is $\mathrm{D}_{\Sigma}(1)$, and $I_{k}=\bigcup\left(I_{k} \cap Y_{r, s}\right)$, so there exist $r_{k}, s_{k}$ such that $I_{k} \cap Y_{r_{k}, s_{k}}$ has interior. By 1.9.(2), there exist $a, b>0$ such that each $Y_{r_{k}, s_{k}} \subseteq Y_{a, b}$, so $I_{k} \cap Y_{a, b}$ has interior for $k=1, \ldots, 2 N+1$. For each $k$, if neither $I_{k}$ nor $I_{k+1}$ is contained in $Y_{a, b}$, then $I_{k} \cup I_{k+1}$ contains at least one of the $N$ closed intervals contained in $Y_{a, b}$. It follows that $Y_{a, b}$ contains some $I_{k}$.

We now characterize the $\mathrm{D}_{\Sigma}(1)$ sets.

3.2. A unary set is $\mathrm{D}_{\Sigma}$ if and only if it is a finite union of points and intervals if and only if it is constructible and definable.

Proof. Let $A \subseteq R$ be definable. Suppose that $A$ is $\mathrm{D}_{\Sigma}$. On the one hand, $\operatorname{int}(A)$ is open and definable, and thus a finite union of intervals by Lemma 2.5. On the other hand, $A \backslash \operatorname{int}(A)$ is $\mathrm{D}_{\Sigma}$ by [1.10, and thus is finite by 3.1. Hence, $A$ is a finite union of points and intervals. Of course, finite unions of points and intervals are constructible and definable, and constructible definable sets are $\mathrm{D}_{\Sigma}$ by 1.10

Definition. Let $\Pi(n, m)$ denote the collection of all coordinate projection maps

$$
\left(x_{1}, \ldots, x_{n}\right) \mapsto\left(x_{\lambda(1)}, \ldots, x_{\lambda(m)}\right): R^{n} \rightarrow R^{m},
$$

where $\lambda:\{1, \ldots, m\} \rightarrow\{1, \ldots, n\}$ is a strictly increasing function. The dimension of a nonempty set $A \subseteq R^{n}$, denoted by $\operatorname{dim} A$, is the largest integer $d$ such that $\pi A$ has interior for some $\pi \in \Pi(n, d)$. We also set $\operatorname{dim} \emptyset=-\infty$ and $\Pi(n,-\infty)=$ $\emptyset=R^{-\infty}$. Notice that $\operatorname{dim} A=d \geq 0$ if and only if, after some permutation of coordinates, the projection of $A$ onto the first $d$ coordinates has interior. The full dimension of $A \subseteq R^{n}$, denoted by $\operatorname{fdim} A$, is the lexicographically ordered pair $(d, k)$, where $d=\operatorname{dim} A$ and $k=\operatorname{card}\{\pi \in \Pi(n, d): \operatorname{int}(\pi A) \neq \emptyset\}$. As $k$ adds no information if $d \in\{-\infty, 0, n\}$, we identify $\operatorname{dim}$ and fdim in these cases.

Next, via 2.2 and 3.2, an easy induction on the dimension of the ambient space yields a characterization of the $\mathrm{D}_{\Sigma}$ sets of dimension 0 .

3.3. A $\mathrm{D}_{\Sigma}$ set has dimension 0 if and only if it is discrete if and only if it is finite.

We are now ready to finish the proof of Theorem A.

3.4. Every $\mathrm{D}_{\Sigma}$ set is constructible.

Proof. We proceed by induction on $n \geq 1$ and $(d, k)=f \operatorname{dim} A$ to show that if $A \in \mathrm{D}_{\Sigma}(n)$, then $A$ is constructible.

The case $A=\emptyset$ is trivial. The cases $n=1$ and $d=0$ are covered by 3.2 and 3.3 ,

Suppose that $0<d=n$. Write $A=\operatorname{int}(A) \cup(A \backslash \operatorname{int}(A))$. By 1.10, $A \backslash \operatorname{int}(A)$ is $\mathrm{D}_{\Sigma}$. By the induction hypothesis on $d$, we have that $A \backslash \operatorname{int}(A)$ is constructible. Then $A$ is constructible. 
We now need deal only with the case $0<d<n$. By permuting coordinates, we may assume further that $\pi A$ has interior, where $\pi$ denotes projection onto the first $d$ coordinates. Write $A=\bigcup X_{r, s}$, where each $X_{r, s}$ is CBD. Put $S=\left\{x \in R^{d}\right.$ : $\left.\operatorname{dim} A_{x}>0\right\}$. As

$$
S=\bigcup_{\rho \in \Pi(n-d, 1)} \bigcup_{r, s>0}\left\{x \in R^{d}:(\exists t \in R)[t, t+s] \subseteq \rho\left(X_{r, s, x}\right)\right\},
$$

we apply 1.9(1), 2.8(3), and the induction hypothesis on $n$ to conclude that $S$ is constructible. By combining 2.8](2), 2.8(3), and 3.1] with $\operatorname{dim} A=d$, we see that $S$ has no interior, and so is nowhere dense by 1.1](3). The induction hypothesis on fdim then yields that $A \cap \pi^{-1} S$ is constructible. Hence, by 1.10](3), we reduce to the case that $S=\emptyset$, that is, $\operatorname{dim} A_{x}=0$ for all $x \in \pi A$. By 3.3 and UF, there exists $N \in \mathbb{N}$ such that card $A_{x} \leq N$ for every $x \in R^{d}$. Let $U$ be the set of all $x \in R^{d}$ such that for some $\epsilon>0$ and positive integer $M$ there exist continuous maps $h_{1}, \ldots, h_{M}: B(x, \epsilon) \rightarrow R^{n-d}$ such that

$$
A \cap \pi^{-1}(B(x, \epsilon))=\bigcup_{i=1}^{M} \operatorname{graph}\left(h_{i}\right)
$$

Observe that $U$ is open by definition. For each fixed $M \in \mathbb{N}$, the set $A(M)$ of all $x \in R^{d}$ such that card $A_{x} \leq M$ is definable. Hence, there are definable functions $g_{1} \leq \cdots \leq g_{M}$ such that $A_{x}=\left\{g_{1}(x), \ldots, g_{M}(x)\right\}$ for every $x \in A(M)$. The interior of $A(M)$ is definable, as is the set of all points such that $\left(g_{1}, \ldots, g_{M}\right)$ is continuous on a box about $x$. As card $A_{x} \leq N$ for every $x \in R^{d}$, we may take $M$ to range only over $\{1, \ldots, N\}$. Hence, $U$ is also definable. By 1.1(5), $A \cap \pi^{-1} U$ is locally closed, hence constructible, so we now need only show that $A \backslash \pi^{-1} U$ is constructible. As $A$ is $\mathrm{D}_{\Sigma}$ and $\pi^{-1} U$ is open, we have by $1.10(3)$ that $A \backslash \pi^{-1} U$ is $\mathrm{D}_{\Sigma}$. By the induction hypothesis on fdim, it suffices now to show that $\pi A \backslash U$ has no interior. For this, we show by induction on $N \geq 1$ that $U$ is dense in the interior of $\pi A$.

Suppose that $N=1$. Define $f: \pi A \rightarrow R^{n-d}$ by letting $f(x)$ be the unique element of $A_{x}$. Then $A=\operatorname{graph}(f)$. Let $B \subseteq \pi A$ be a closed box. As graph $(f\lceil B)$ is $\mathrm{D}_{\Sigma}$, we have

$$
\operatorname{graph}\left(f\lceil B)=\bigcup X_{r, s}=\bigcup \operatorname{graph}\left(f\left\lceil\pi\left(X_{r, s}\right)\right),\right.\right.
$$

where each $X_{r, s}$ is CBD. By 2.3, each $f\left\lceil\pi\left(X_{r, s}\right)\right.$ is continuous. By 3.1, there exist $r, s>0$ such that $\pi\left(X_{r, s}\right)$ contains a box $B^{\prime}$. Then $B^{\prime} \subseteq U$, as required.

Now suppose that $N>1$ and let $B \subseteq \pi A$ be a box. Write $A \cap \pi^{-1} B=$ $\bigcup_{r, s>0} X_{r, s}$, where each $X_{r, s}$ is CBD. By 3.1. there exist $r, s>0$ such that $\pi\left(X_{r, s}\right)$ contains a closed bounded box $D$. Define $f: D \rightarrow R^{n-d}$ by $f(x)=\operatorname{lexmin} X_{r, s, x}$. By combining the inductive assumption on $n$ with 1.10, (1), 2.8 (1) and 3.1) we have that $\mathcal{D}(f)$ is nowhere dense, so there is a box $B^{\prime} \subseteq D$ such that $f\left\lceil B^{\prime}\right.$ is continuous. If $A \cap \pi^{-1} B^{\prime}=\operatorname{graph}\left(f\left\lceil B^{\prime}\right)\right.$, then $B^{\prime} \subseteq U$, and we are done. If not, then by applying the induction hypothesis on $N$ to $\left(A \cap \pi^{-1} B^{\prime}\right) \backslash \operatorname{graph}(f)$, we obtain a box $B^{\prime \prime} \subseteq B^{\prime}, M \geq 1$ and continuous functions $h_{1}, \ldots, h_{M}: B^{\prime \prime} \rightarrow R^{n-d}$ such that

$$
\left(A \cap \pi^{-1} B^{\prime \prime}\right) \backslash \operatorname{graph}(f)=\bigcup_{i=1}^{M} \operatorname{graph}\left(h_{i}\right),
$$

so $B^{\prime \prime} \subseteq U$. In either case, $U \cap B$ has interior. 
We have shown that $U$ is dense $\operatorname{in} \operatorname{int}(\pi A)$, thus ending the proof of [3.4, as well as the proof of Theorem A.

We close this section with a corollary of the proof of Theorem A.

3.5 (Analogue of Baire Category Theorem). Let $Y \subseteq R^{>0} \times R^{>0} \times R^{n}$ be definable such that each $Y_{r, s}$ is nowhere dense and $Y_{r, s} \subseteq Y_{r^{\prime}, s^{\prime}}$ whenever $r \leq r^{\prime}$ and $s^{\prime} \leq s$. Then $\bigcup Y_{r, s}$ is contained in a finite union of nonopen cells (and thus is nowhere dense).

Proof. For $r, s>0$, put $X_{r, s}=\operatorname{cl}\left(Y_{r, s}\right) \cap[-r, r]^{n}$. Then $\left(X_{r, s}\right)$ is $\mathrm{D}_{\Sigma}$ and $\bigcup Y_{r, s} \subseteq$ $\bigcup X_{r, s}$. Each $X_{r, s}$ has no interior, so the same is true of $\bigcup X_{r, s}$ by 3.1. We have shown that $\mathfrak{R}^{\circ}$ is o-minimal and is interdefinable with the expansion of $R$ by its $\mathrm{D}_{\Sigma}$ sets, so $\bigcup X_{r, s}$ is a finite union of nonopen cells.

Having established Theorem A, we have achieved the first main goal of this paper: a reasonable generalization of 2.14 that does not rely on working over $\mathbb{R}$. In the next section, we develop some general model theory associated with open cores.

\section{OPEN CORE BASICS}

In this section, we develop a number of general results relating structures with their open cores, particularly when the open core is o-minimal. The latter part of the section concentrates on the NDG setting. Before proceeding, the reader may wish to read any parts of Sections 1 and 2 that were skipped earlier. We begin with some easy facts.

4.1. (1) Every constructible set definable in $\mathfrak{R}$ is definable in $\mathfrak{R}^{\circ}$.

(2) The cells of $\mathfrak{R}$ are exactly those of $\mathfrak{R}^{\circ}$.

(3) Every set definable in an o-minimal reduct of $\mathfrak{R}$ is definable in $\mathfrak{R}^{\circ}$.

(4) If $\mathfrak{R}^{\circ}$ is o-minimal, then $\mathfrak{R} \vDash \mathrm{DC}$.

N.B. Items (1) and (2) relieve us of having to distinguish between $\mathfrak{R}$ and $\mathfrak{R}^{\circ}$ when talking about cells, decompositions, and definable constructible sets.

Proof. Item (1) is immediate from [1.4 Cells are locally closed, so (2) follows from (1). In turn, (3) follows (2) and cell decomposition. For (4), let $\mathfrak{R}^{\circ}$ be o-minimal and $A \subseteq R$ be definable. Then $\operatorname{cl}(A)$ is a union of finitely many points and closed intervals, so both $\inf \operatorname{cl}(A)$ and $\sup \operatorname{cl}(A)$ exist in $R \cup\{ \pm \infty\}$. As $\sup A=$ $\sup \operatorname{cl}(A)$ and $\inf A=\inf \operatorname{cl}(A)$, we have $\inf A, \sup A \in R \cup\{ \pm \infty\}$. Hence, $\mathfrak{R} \vDash$ DC.

The so-called tame geometry, topology, and model theory of o-minimal structures derives in great measure from cell decomposition and its refinements. Although a structure whose open core is o-minimal but which is not itself o-minimal does not enjoy full cell decomposition, it does satisfy a weaker form that suggests that there is a notion of dimension for definable sets that is controlled by the o-minimal open core, especially if the structure also satisfies NDG (see 4.15) and Theorem 4.16).

Definition. Given a topological space $X$ and collections $\mathcal{Y}, \mathcal{Z}$ of subsets of $X$, we say that $\mathcal{Y}$ is quasicompatible with $\mathcal{Z}$ if for every $Y \in \mathcal{Y}$ and $Z \in \mathcal{Z}$ such that $Y \cap Z \neq \emptyset$, we have either $Y \subseteq Z$ or $Z$ dense and co-dense in $Y$. (Recall that $\mathcal{Y}$ is compatible with $\mathcal{Z}$ if the last alternative is omitted.) 
Loosely, the idea is that $\mathcal{Y}$ can be used to refine $\mathcal{Z}$ except possibly for a certain amount of noise that cannot be avoided, but at least can be organized by $\mathcal{Y}$. The next result, established in [26, §5] and [28, p. 203], makes this idea precise.

Quasidecomposition. $\mathfrak{R}^{\circ}$ is o-minimal if and only if for every finite collection $\mathcal{A}$ of subsets of $R^{n}$ that are definable in $\mathfrak{R}$ there is a decomposition $\mathcal{C}$ of $R^{n}$ that is quasicompatible with $\mathcal{A}$.

Many of the usual variations that hold in the o-minimal setting have analogues here as well. The cells in $\mathcal{C}$ can be taken to be defined over the same set of parameters as the sets in $\mathcal{A}$. If $\mathfrak{R}$ expands an ordered field, then the cells in $\mathcal{C}$ can be taken to be $C^{p}$ for each fixed $p \in \mathbb{N}$. If $\Re$ has $C^{\infty}$ cell decomposition, then $\mathcal{C}$ can be chosen so that each $C \in \mathcal{C}$ is a $C^{\infty}$ cell, and so on. Many results from o-minimality thus transfer immediately modulo obvious modifications if the open core is o-minimal. We shall not dwell in this paper on such applications. On the other hand, caution is in order. In contrast to the o-minimal setting, quasidecomposition need not be uniform in parameters; that is, it may be impossible to choose $\mathcal{C}$ so that $\mathcal{C}_{x}$ is quasicompatible with $\mathcal{A}_{x}$ for every $x \in R^{m}$ and $m \leq n$. Indeed, we shall see below (4.16) that this uniform version holds if and only if $\mathfrak{R}^{\circ}$ is o-minimal and $\mathfrak{R} \vDash$ NDG.

Elementarity. Heretofore, we have confined our attention primarily to definability theory over the fixed structure $\mathfrak{R}$. We now begin to deal with more general model-theoretic concerns. In particular, given $T \supseteq \mathrm{DLO}$, we are interested in understanding how $\mathfrak{M}^{\circ}$ varies as $\mathfrak{M}$ ranges over models of $T$. A natural first question along theses lines is whether the property of having o-minimal open core is elementary. We do not yet know if this true, but the next result clarifies the issue.

4.2. (1) If $\mathfrak{R}^{\circ}$ is locally o-minimal and $\mathfrak{R} \vDash \mathrm{DC}$, then $\mathfrak{M}^{\circ}$ is locally o-minimal for every $\mathfrak{M} \equiv \mathfrak{R}$.

(2) If $\mathfrak{R}^{\circ}$ is o-minimal, then $\mathfrak{M}^{\circ}$ is locally o-minimal for every $\mathfrak{M} \equiv \mathfrak{R}$.

(3) If $\mathfrak{R}^{\circ}$ is o-minimal and $\mathfrak{R} \vDash \mathrm{UF}$, then $\mathfrak{M}^{\circ}$ is o-minimal for every $\mathfrak{M} \equiv \mathfrak{R}$.

(4) If $\mathfrak{M}^{\circ}$ is weakly o-minimal for every $\mathfrak{M} \equiv \mathfrak{R}$, then $\mathfrak{R} \vDash \mathrm{UF}$.

(5) $\mathfrak{M}^{\circ}$ is o-minimal for every $\mathfrak{M} \equiv \mathfrak{R}$ if and only if $\mathfrak{R}^{\circ}$ is o-minimal and $\mathfrak{R} \vDash \mathrm{UF}$.

Proof. (1). As DC is elementary, it suffices by 2.11 to show that if $A \subseteq M$ is definable in $\mathfrak{M}^{\circ}$, then $A$ either has interior or is closed and discrete. Now, $A$ is defined in $\mathfrak{M}$ by a formula of the form

$$
\psi\left(p, y_{n+1}\right):=\mathrm{Q} y_{1} \ldots \mathrm{Q} y_{n} \varphi\left(p, y_{1} \ldots, y_{n+1}\right),
$$

where each $\mathrm{Q}_{i}$ is a quantifier, $\varphi$ is an $(m+n+1)$-ary formula in the language of $\mathfrak{R}$ (without parameters), $p \in M^{m}$ and the set $\varphi\left(p, M^{n+1}\right)$ is constructible. By 1.1 , there exists $N \in \mathbb{N}$ such that $\varphi\left(p, M^{n+1}\right)$ is a union of $N$ locally closed sets. By 1.4. the set

$$
\left\{q \in M^{m}: \varphi\left(q, M^{n+1}\right) \text { is a union of } N \text { locally closed sets }\right\}
$$

is $\emptyset$-definable in $\mathfrak{M}$, so

$$
X:=\left\{x \in R^{m}: \varphi\left(x, R^{n+1}\right) \text { is a union of } N \text { locally closed sets }\right\}
$$

is $\emptyset$-definable in $\mathfrak{R}$. Then for each $x \in X, \psi(x, R)$ is definable in $\mathfrak{R}^{\circ}$, and thus either has interior or is closed and discrete. As this property is elementary, $\psi(p, M)$ $(=A)$ either has interior or is closed and discrete. 
Now, (2) follows from 4.1(4) and (1), while (3) follows from (2) and 2.12. For (4), recall 1.16, and (5) is immediate from (3) and (4).

Specializing to sets of parameters. Recall that our definition of $\mathfrak{R}^{\circ}$ included specification of the language of $\mathfrak{R}^{\circ}$, namely, a relation symbol for each nonempty open set definable in $\mathfrak{R}$. This was done for concreteness, but it can be cumbersome in practice. If $\mathfrak{R}$ is an expansion of $(R,<)$ by constructible sets, then $\mathfrak{R}^{\circ}=\mathrm{df}_{\mathrm{df}} \mathfrak{R}$ by 1.4, but obviously the language of $\Re$ may be much more convenient than that of $\mathfrak{R}^{\circ}$. For example, every real semilinear open set is definable in $(\mathbb{R},<,+)$, every real semialgebraic open set is definable in $(\mathbb{R},+, \cdot)$, and every real open set is definable in $(\mathbb{R},+, \cdot, \mathbb{Z})$. This is one motivation for our notion "open core of a theory". More substantial: By definition, the language of $\mathfrak{R}^{\circ}$ contains a unary relation symbol for each open set $R \backslash\{r\}$ with $r \in R$. Hence, every element of $R$ is $\emptyset$-definable in $\mathfrak{R}^{\circ}$, and the languages of the open cores vary as we range over elementarily equivalent structures. These features of the definition are harmless if all we care about is definability in $\mathfrak{R}^{\circ}$, but certainly they are antithetical to more general model-theoretic concerns. We now attempt to ameliorate the situation.

Given $S \subseteq R$, let $\mathfrak{R}_{S}^{\circ}$ denote the structure $(R,(U))$, where $U$ ranges over the open sets, of all arities, that are $S$-definable in $\mathfrak{R}$. For concreteness, we regard $\mathfrak{R}_{S}^{\circ}$ as a structure in a language consisting precisely of relation symbols for the nonempty open sets that are $S$-definable in $\mathfrak{R}$. For $S=R$, we continue to write just $\mathfrak{R}^{\circ}$. As per our discussion above, we shall be interested primarily in the cases $S=R$ and $S=\emptyset$, and how they are related. Nevertheless, for illustrative purposes, we start out with a few easy results for arbitrary $S \subseteq R$.

Clearly, for every $A, B \subseteq R$, every set that is $B$-definable in $\mathfrak{R}_{A}^{\circ}$ is $\emptyset$-definable in $\mathfrak{R}_{A \cup B}^{\circ}$. On the other hand, if $A \subsetneq B$ and $U$ is open and $B$-definable in $\mathfrak{R}$, then it is not at all clear as to whether $U$ is definable in $\mathfrak{R}_{A}^{\circ}$ or not, even if $\mathfrak{R}^{\circ}$ is o-minimal. This issue arises in several ways in the sequel.

We easily obtain (possibly weaker) versions of 4.1 , quasidecomposition, and Theorem A:

4.3. Let $S \subseteq R$.

(1) (a) Every constructible set $S$-definable in $\mathfrak{R}$ is $\emptyset$-definable in $\mathfrak{R}_{S}^{\circ}$.

(b) The $S$-definable cells of $\mathfrak{R}$ are exactly the $\emptyset$-definable cells of $\mathfrak{R}_{S}^{\circ}$.

(c) Every set definable in an o-minimal reduct of $\mathfrak{R}$ is definable in $\mathfrak{R}_{\emptyset}^{\circ}$.

(d) If $\Re_{S}^{\circ}$ is o-minimal, then both $\inf A$ and $\sup A$ exist in $R \cup\{ \pm \infty\}$ for every $S$-definable $A \subseteq R$.

(2) If $\mathfrak{R}_{S}^{\circ}$ is o-minimal, then for every finite collection $\mathcal{A}$ of subsets of $R^{n}$ that are $S$-definable in $\mathfrak{R}$, there is a decomposition $\mathcal{C}$ of $R^{n}$ into $S$-definable cells such that $\mathcal{C}$ is quasicompatible with $\mathcal{A}$.

(3) If $\mathfrak{R} \vDash \mathrm{UF}$ and expands a densely ordered group, and $\sup A$ exists in $R \cup$ $\{ \pm \infty\}$ for every $S$-definable unary set, then $\mathfrak{R}_{S}^{\circ}$ is o-minimal.

Proof. (1). Again, (a) is immediate from 1.4, while (b) follows from (a), and (d) is an easy modification of the proof of 4.1(4). For (c), let $A \subseteq R^{n}$ be definable in some o-minimal reduct of $\mathfrak{R}$. Then $A=B_{x}$ for some $m \in \mathbb{N}, x \in R^{m}$ and $B \subseteq R^{m+n}$ $\emptyset$-definable in $\mathfrak{R}$ such that $(R,<, B)$ is o-minimal. As there is a decomposition of $R^{m+n}$ into $\emptyset$-definable cells compatible with $B$, we have $B$ definable in $\mathfrak{R}_{\emptyset}^{\circ}$. Hence, $A$ is definable in $\Re_{\emptyset}^{\circ}$.

(2). See the proof of the corresponding part of quasidecomposition [28, p. 203]. 
(3). By the group structure, $\inf A$ and $\sup A$ then exist in $R \cup\{ \pm \infty\}$ for every unary set $S$-definable in $\mathfrak{R}$. Hence, $\inf A$ and $\sup A$ exist in $R \cup\{ \pm \infty\}$ for every unary set $\emptyset$-definable in $\left(\Re,(s)_{s \in S}\right)$. By replacing "definable" with " $\emptyset$-definable" throughout the proof of Theorem A, every unary set $\emptyset$-definable in $\mathfrak{R}_{S}^{\circ}$ is a finite union of points and intervals. By 1.14, $\mathfrak{R}_{S}^{\circ}$ is o-minimal.

Note. In contrast to the case $S=R$, we do not know if o-minimality of $\mathfrak{R}_{S}^{\circ}$ implies that $\mathfrak{R} \vDash \mathrm{DC}$, if o-minimality of $\mathfrak{R}_{S}^{\circ} \vDash$ UF implies that $\mathfrak{R} \vDash \mathrm{UF}$, nor if the converse of (2) is true.

Open cores of theories. With the introduction of $\mathfrak{R}_{\emptyset}^{\circ}$, we now have a fixed language canonically associated to $\mathfrak{R}$, the language of $\mathfrak{R}_{\emptyset}^{\circ}$, in which we can work over all models of $\operatorname{Th}(\mathfrak{R})$. Now, we certainly have $\mathfrak{M}_{\emptyset}^{\circ} \equiv \mathfrak{R}_{\emptyset}^{\circ}$ for every $\mathfrak{M} \equiv \mathfrak{R}$, but this does not help us understand $\mathfrak{M}^{\circ}$ unless we also understand the relationship between $\mathfrak{M}^{\circ}$ and $\mathfrak{M}_{\emptyset}^{\circ}$. Our next result provides further motivation for our notion of open cores of theories.

4.4. The following are equivalent.

(1) $\mathfrak{M}^{\circ}={ }_{\text {df }} \mathfrak{M}_{\emptyset}^{\circ}$ for every $\mathfrak{M} \equiv \mathfrak{R}$.

(2) $\operatorname{Th}\left(\mathfrak{R}_{\emptyset}^{\circ}\right)$ is an open core of $\operatorname{Th}(\mathfrak{R})$.

(3) For every $B \subseteq R^{m+n} \emptyset$-definable in $\mathfrak{R}$ there exist $k \in \mathbb{N}$ and $C \subseteq R^{k+n}$ $\emptyset$-definable in $\Re_{\emptyset}^{\circ}$ such that

$$
\left\{B_{x}: x \in R^{m} \& B_{x} \text { is open }\right\} \subseteq\left\{C_{y}: y \in R^{k}\right\} .
$$

Proof. $(1) \Rightarrow(2)$ holds by definition. For $(2) \Rightarrow(3)$, extend the language of $\mathfrak{R}$ by symbols for the $\emptyset$-definable open sets and apply compactness. For $(3) \Rightarrow(1)$, let $\mathfrak{M} \equiv \mathfrak{R}$ and $U \subseteq M^{n}$ be open and definable in $\mathfrak{M}$, say, $U=B_{x}^{\prime}$ for some $m \in \mathbb{N}$, $x \in M^{m}$ and $B^{\prime} \subseteq M^{m+n} \emptyset$-definable in $\mathfrak{M}$. Let $B$ be the set defined in $\mathfrak{R}$ by the formula that defines $B^{\prime}$ in $\mathfrak{M}$, let $C$ be as in (3), and let $C^{\prime}$ be the set defined in $\mathfrak{M}_{\emptyset}^{\circ}$ by the formula that defines $C$ in $\mathfrak{R}_{\emptyset}^{\circ}$. As $\mathfrak{M}_{\emptyset}^{\circ} \equiv \mathfrak{R}_{\emptyset}^{\circ}$, there exists $y \in M^{k}$ such that $C_{y}^{\prime}=B_{x}^{\prime}=U$. Hence, $U$ is definable in $\mathfrak{M}_{\emptyset}^{\circ}$.

4.5. If every set $\emptyset$-definable in $\mathfrak{R}$ is constructible, then $\operatorname{Th}\left(\mathfrak{R}_{\emptyset}^{\circ}\right)$ is an open core of $\operatorname{Th}(\mathfrak{R})$.

Proof. Let $\mathfrak{M} \equiv \mathfrak{R}$ and $A^{\prime} \subseteq M^{n}$ be definable in $\mathfrak{M}$; say, $A^{\prime}=B_{x}^{\prime}$ for some $m \in \mathbb{N}, x \in M^{m}$, and $B^{\prime} \subseteq M^{m+n}$ that is $\emptyset$-definable in $\mathfrak{M}$. Let $B$ be the subset of $R^{m+n}$ defined in $\mathfrak{R}$ by the formula that defines $B^{\prime}$ in $\mathfrak{M}$. By assumption, $B$ is constructible. By 4.3(1), $B$ is $\emptyset$-definable in $\mathfrak{R}_{\emptyset}^{\circ}$. As $\mathfrak{R}_{\emptyset}^{\circ} \equiv \mathfrak{M}_{\emptyset}^{\circ}$, we have that $B^{\prime}$ is definable in $\mathfrak{M}_{\emptyset}^{\circ}$, hence so is $A^{\prime}$. We have shown that $\mathfrak{M}^{\circ}={ }_{\mathrm{df}} \mathfrak{M}_{\emptyset}^{\circ}$. Now apply 4.4

Atomic models. As mentioned previously (1.12), every complete o-minimal theory has a prime model that is unique up to isomorphism. Furthermore this prime model is also atomic. (Of course, for every complete theory in a countable language, countable atomic and prime models coincide and are unique up to isomorphism.) We shall see in Sections 5 and 6 that the situation is more complicated in the setting of o-minimal open cores. First, observe that every structure $\mathfrak{M}$ such that $M=\operatorname{dcl}(\emptyset)$ is atomic. (If $x \in M^{n}$, then the formula that defines $x$ is complete with respect to $\mathrm{Th}(\mathfrak{M})$.) We shall need a partial converse.

4.6. If $\mathfrak{R}$ is atomic, $\mathfrak{R}_{\emptyset}^{\circ}$ is o-minimal, and $\left(\operatorname{dcl}_{\mathfrak{R}}(\emptyset),<\right) \vDash \mathrm{DLO}$, then $R=\operatorname{dcl}_{\mathfrak{R}}(\emptyset)$. 
Proof. Let $r \in R$. As $\mathfrak{R}$ is atomic, $r$ satisfies a unary formula $\varphi$ that is complete with respect to $\operatorname{Th}(\mathfrak{R})$. It suffices to show that $\varphi(\mathfrak{R})$ is finite. Suppose not. By quasidecomposition, $\varphi(\Re)$ is dense in some $\emptyset$-definable open interval $(x, y)$ with $x, y \in R \cup\{ \pm \infty\}$. By assumption, there exists a $\emptyset$-definable $z$ such that $x<z<y$. Let $\psi$ be the formula defining the ray $(z, \infty)$. Then both $\varphi \wedge \psi$ and $\varphi \wedge \neg \psi$ are consistent with $\operatorname{Th}(\Re)$, contradicting that $\varphi$ is complete.

Thus, by our convention that ordered groups come equipped with $1>0$,

4.7. If $\mathfrak{R}$ expands a densely ordered group and $\mathfrak{R}_{\emptyset}^{\circ}$ is o-minimal, then $\mathfrak{R}$ is atomic if and only if $R=\mathrm{dcl}(\emptyset)$.

Preservation of open cores. Given an expansion $\widehat{\mathfrak{R}}$ of $\mathfrak{R}$, we wish to find conditions guaranteeing that $\widehat{\mathfrak{R}}^{\circ}=\mathfrak{R}^{\circ}$, especially if $\mathfrak{R}^{\circ}$ is known to be o-minimal. $\left(\widehat{\mathfrak{R}}^{\circ}\right.$ denotes the open core of $\widehat{\Re}$.) As usual, we are interested primarily in the group case, but we work in greater generality to some extent. We begin with a technical lemma that is somewhat stronger than what we need here, but may prove useful in future investigations. Recall from 1.19 the definition of "given piecewise".

4.8. Suppose that $\mathfrak{R}^{\circ}$ is o-minimal and $\widehat{\mathfrak{R}} \vDash$ DC. Let $C$ be a cell in $\mathfrak{R}$ and $f: C \rightarrow R$ be continuous, definable in $\widehat{\Re}$, and given piecewise by functions definable in $\mathfrak{\Re}$. Then $f$ is definable in $\mathfrak{R}$.

Proof. By hypothesis, there exist finitely many functions $g_{1}, \ldots, g_{N}: C \rightarrow R$ definable in $\mathfrak{R}$ such that $\operatorname{graph}(f) \subseteq \bigcup_{i=1}^{N} \operatorname{graph}\left(g_{i}\right)$. We now proceed by induction on $N \geq 1$. The case $N=1$ is trivial. Let $N>1$ and assume the result for all $k<N$. By replacing $g_{1}$ with $\min \left(g_{1}, \ldots, g_{N}\right)$, and so on, we suppose that $g_{1} \leq \cdots \leq g_{N}$. To simplify notation, we write $g_{i}^{+}$instead of $\lim \sup g_{i}$ and $g_{i}^{-}$instead of liminf $g_{i}$, both of which exist in $R \cup\{ \pm \infty\}$ by DC. The sets

$$
\left\{(x, t) \in \operatorname{graph}\left(g_{i}\right): t<g_{i}^{-}(x)\right\}, \quad\left\{(x, t) \in \operatorname{graph}\left(g_{i}\right): g_{i}^{+}(x)<t\right\}
$$

are discrete and definable in $\mathfrak{R}$, hence finite. Thus, for all $i$, we may take $g_{i}^{-} \leq g_{i} \leq$ $g_{i}^{+}$(on all of $C$ ). For $1 \leq i<j \leq N$, the sets $\left\{x \in C: g_{i}^{+}(x)<g_{j}^{-}(x)\right\}$ are open in $C$, hence definable in $\mathfrak{R}^{\circ}$. Thus, we reduce to the case that for every $1 \leq i<j \leq N$, we have either $g_{i}^{+}<g_{j}^{-}$or $g_{i}^{+} \geq g_{j}^{-}$. If $g_{1}^{+} \geq g_{N}^{-}$, then $g_{1}^{+}=f=g_{N}^{-}$by continuity of $f$, and we are done. On the other hand, if $g_{1}^{+}<g_{N}^{-}$, then we may choose $i<j$ such that $g_{i}^{+}<g_{j}^{-}$and $j-i$ is minimal. Put $A=\left\{x \in C: f(x)<g_{j}^{-}(x)\right\}$ and $B=\left\{x \in C: g_{i}^{+}(x)<f(x)\right\}$. Then $f \leq g_{j-1}$ on $A, f \geq g_{i+1}$ on $B$, and $C=A \cup B$. By continuity of $f$, both $A$ and $B$ are open in $C$. By DC, cells are definably connected in $\widehat{\Re}(2.9)$, so it suffices by the inductive assumption to show that $A \cap B=\emptyset$. This is immediate if $i+1=j$ (recall that $g_{i} \leq g_{i}^{+}$and $g_{i+1}^{-} \leq g_{i+1}$ ), so suppose that $i+1<j$. Then $g_{j-1}^{-} \leq g_{i}^{+}<g_{j}^{-} \leq g_{i+1}^{+}$by minimality of $j-i$. But on $A \cap B$, we have $g_{i+1} \leq f \leq g_{j-1}$, hence also $g_{i+1}^{+} \leq g_{j-1}^{-}$by continuity of $f$.

The next two results are immediate consequences.

4.9. If $\widehat{\mathfrak{R}}^{\circ}$ is o-minimal and every function definable in $\widehat{\mathfrak{R}}^{\circ}$ is given piecewise by functions definable in $\mathfrak{R}$, then $\widehat{\mathfrak{R}}^{\circ}=\mathfrak{R}^{\circ}$.

4.10. If $\mathfrak{R}^{\circ}$ is o-minimal and every function definable in $\mathfrak{R}^{\circ}$ is given piecewise by functions definable in $\mathfrak{R}_{\emptyset}^{\circ}$, then $\mathfrak{R}^{\circ}={ }_{\mathrm{df}} \mathfrak{R}_{\emptyset}^{\circ}$. 
The next result will be used in Section 6 .

4.11. Suppose that $\widehat{\mathfrak{R}} \vDash \mathrm{UF}$, every unary open set definable in $\widehat{\mathfrak{R}}$ is a finite union of open intervals, every function definable in $\widehat{\Re}$ is given piecewise by functions definable in $\mathfrak{R}$, and $\mathfrak{R}^{\circ}$ is o-minimal. Then $\widehat{\mathfrak{R}}^{\circ}=\mathfrak{R}^{\circ}$.

Proof. By quasidecomposition and 4.9, it suffices to show that given $A_{1}, \ldots, A_{k} \subseteq$ $R^{m+1}$ definable in $\widehat{\Re}$ there is a decomposition relative to $\widehat{\Re}$ of $R^{m+1}$ that is quasicompatible with $A_{1}, \ldots, A_{k}$. The argument is quite similar to parts of the proof of cell decomposition in the o-minimal case, so we provide only a sketch. Indeed, refer to [10, p. 55]; we indicate the needed modifications. Change the definition of $\operatorname{bd}_{m}(A)$ to $\left\{(x, r) \in R^{m+1}: r \in \operatorname{bd}\left(\operatorname{cl}\left(A_{x}\right)\right) \cup \operatorname{bd}\left(\operatorname{int}\left(A_{x}\right)\right)\right\}$. Observe that if $A$ is definable in $\widehat{\Re}$, then $\operatorname{int}\left(A_{x}\right)$ is a finite union of open intervals, and $\operatorname{cl}\left(A_{x}\right)$ is a finite union of points and closed intervals. Let $\mathcal{G}$ be a finite collection of functions $R^{m} \rightarrow R$ definable in $\mathfrak{R}$ such that every $f_{i j}$ is given piecewise by $\mathcal{G}$. By quasidecomposition and o-minimality of $\mathfrak{R}^{\circ}$, there is a decomposition $\mathcal{C}$ of $R^{m+1}$ quasicompatible with $\{\operatorname{graph}(g): g \in \mathcal{G}\}$. For all $g, h \in \mathcal{G}$, the sets

$$
\begin{array}{cll}
\left\{x \in B_{i}: f_{i j}(x)<g(x)\right\}, & \left\{x \in B_{i}: f_{i j}(x)=g(x)\right\}, & \left\{x \in B_{i}: f_{i j}(x)>g(x)\right\}, \\
\left\{x \in R^{m}: g(x)<h(x)\right\}, & \left\{x \in R^{m}: g(x)=h(x)\right\}, & \left\{x \in R^{m}: g(x)>h(x)\right\}
\end{array}
$$

are definable in $\widehat{\mathfrak{R}}$, as are the sets of $x \in B_{i}$ such that $\left(A_{\lambda}\right)_{x}$ is dense and co-dense in $(g(x), h(x))$. The rest of the modification is routine.

We now focus on the group case, beginning with an easy observation.

4.12. If $\widehat{\mathfrak{R}} \vDash \mathrm{DC}$ and expands a densely ordered group, then $\widehat{\mathfrak{R}}^{\circ}=\mathfrak{R}^{\circ}$ if and only if every continuous function definable in $\widehat{\mathfrak{R}}$ is definable in $\mathfrak{R}$.

Proof. Every closed set $A$ definable in $\widehat{\Re}$ is the zero set of the distance function to $A$ (which exists by DC).

Under additional technical assumptions, we may reduce to a condition on unary definable functions:

4.13. Suppose that $\mathfrak{R}$ expands a densely ordered group, $\widehat{\mathfrak{R}}^{\circ}$ is o-minimal and $\aleph_{0}$-saturated, and every cofinitely continuous (that is, continuous off a finite set) unary function definable in $\widehat{\mathfrak{R}}$ is given piecewise by functions definable in $\mathfrak{R}$. Then $\widehat{\mathfrak{R}}^{\circ}=\mathfrak{R}^{\circ}$.

Proof. Then $\widehat{\mathfrak{R}} \vDash \mathrm{DC}$ and $\mathfrak{R}^{\circ}$ is o-minimal. By 4.8, every unary function definable in $\widehat{\mathfrak{R}}^{\circ}$ is definable in $\mathfrak{R}^{\circ}$. As $\aleph_{0}$-saturated o-minimal expansions of ordered groups are determined by their unary definable functions [9, Lemma 4.7], we have $\widehat{\mathfrak{R}}^{\circ}=$ $\mathfrak{R}^{\circ}$.

Caveat. Something like the saturation assumption is needed. For each $n$, there are examples where $\mathfrak{R}$ expands the real field, $\widehat{\mathfrak{R}}$ is o-minimal, and every $n$-ary function definable in $\widehat{\mathfrak{R}}$ is definable in $\mathfrak{R}$, yet $\widehat{\mathfrak{R}}$ defines $(n+1)$-ary functions that are not definable in $\Re$. See Randriambololona [38.

Inspired by the proof of [9, Theorem 5], we arrive at our best general result in this direction. 
4.14. Theorem. If DOG $\subseteq T \subseteq \widehat{T}$, then the following are equivalent.

(1) For every $\widehat{\mathfrak{M}} \vDash \widehat{T}, \widehat{\mathfrak{M}}^{\circ}$ is o-minimal and $\widehat{\mathfrak{M}}^{\circ}=\mathfrak{M}^{\circ}$ (where $\mathfrak{M}$ is the reduct of $\widehat{\mathfrak{M}}$ to the language of $T$ ).

(2) For every $\widehat{\mathfrak{M}} \vDash \widehat{T}$, every unary open set definable in $\widehat{\mathfrak{M}}$ is a finite union of intervals, and every cofinitely continuous unary function definable in $\widehat{\mathfrak{M}}$ is given piecewise by functions definable in $\mathfrak{M}$.

(3) $\widehat{T} \vDash \mathrm{DC}+\mathrm{UF}$ and for every $\widehat{\mathfrak{M}} \vDash \widehat{T}$, every cofinitely continuous unary function definable in $\widehat{\mathfrak{M}}$ is given piecewise by functions definable in $\mathfrak{M}$.

Proof. For $(1) \Rightarrow(2)$, recall 1.1(5) and 4.1. That $(2) \Rightarrow(3)$ is by 2.5. Assume (3); we show that (1) holds. By Theorem A, every model of $\widehat{T}$ has o-minimal open core. Let $\widehat{\mathfrak{M}} \vDash \widehat{T}$ and $\mathfrak{M}$ be the reduct of $\widehat{\mathfrak{M}}$ to the language of $T$. We must show that every open set definable in $\widehat{\mathfrak{M}}$ is definable in $\mathfrak{M}$. By passing to an elementary extension, it suffices to consider the case that $\widehat{\mathfrak{M}}$ is $\aleph_{0}$-saturated. Apply 4.13

No dense graphs. We now investigate the connections between NDG and o-minimality of $\mathfrak{R}^{\circ}$.

4.15. Let $\mathfrak{R}^{\circ}$ be o-minimal and $\mathfrak{R} \vDash \mathrm{NDG}$.

(1) dcl in $\mathrm{Th}(\mathfrak{R})$ is the same as in $\mathrm{Th}\left(\mathfrak{R}_{\emptyset}^{\circ}\right)$.

(2) $\operatorname{Th}(\mathfrak{R}) \vDash \mathrm{EP}+\mathrm{UF}$.

(3) $\operatorname{Th}\left(\Re_{\emptyset}^{\circ}\right)$ is an open core of $\operatorname{Th}(\Re)$.

Proof. (1). Trivially, $\mathfrak{R}_{\emptyset}^{\circ}$ is o-minimal. As both NDG and the o-minimality of $\mathfrak{R}_{\emptyset}^{\circ}$ are elementary, it suffices to show that dcl in $\mathfrak{R}$ is the same as in $\mathfrak{R}_{\emptyset}^{\circ}$. Let $S \subseteq R$ and $b \in R$ be $S$-definable in $\Re$. Choose $n$ minimal for which there exist $A \subseteq R^{n}$, a function $f: A \rightarrow R$ that is $\emptyset$-definable in $\mathfrak{R}$, and $a \in A \cap S^{n}$ such that $f(a)=b$. By o-minimality of $\mathfrak{R}_{\emptyset}^{\circ}$, there is a decomposition $\mathcal{C}$ of $R^{n+1}$ into $\emptyset$-definable cells compatible with $\operatorname{cl}(\operatorname{graph}(f))$. Let $C \in \mathcal{C}$ be such that $(a, b) \in C$. By minimality of $n$, the projection $\pi C$ of $C$ onto the first $n$ coordinates is a $\emptyset$-definable open cell. By NDG, we have $C=\operatorname{graph}\left(f\lceil\pi C)\right.$, so $f\left\lceil\pi C\right.$ is $\emptyset$-definable in $\mathfrak{R}_{\emptyset}^{\circ}$. As $a \in \pi C$, we have that $b(=f(a))$ is $S$-definable in $\mathfrak{R}_{\emptyset}^{\circ}$.

Item (2) is immediate from (1), 1.20, and 1.17

(3). Let $\mathfrak{M} \equiv \mathfrak{R}$. By 4.2 and $(2), \mathfrak{M}^{\circ}$ is o-minimal. By (1) and 1.20 , every function definable in $\mathfrak{M}^{\circ}$ is given piecewise by functions definable in $\mathfrak{M}_{\emptyset}^{\circ}$. Of course, $\mathfrak{M}_{\emptyset}^{\circ}$ is also o-minimal, so $\mathfrak{M}^{\circ}={ }_{\mathrm{df}} \mathfrak{M}_{\emptyset}^{\circ}$ by 4.10. Hence, $\operatorname{Th}\left(\mathfrak{R}_{\emptyset}^{\circ}\right)$ is an open core of $\operatorname{Th}(\Re)$.

The next result characterizes the combination of NDG and o-minimality of the open core by NDG for the unary definable functions as well as by uniformity in parameters of quasidecomposition. Definability is with respect to $\mathfrak{R}$ throughout.

4.16. Theorem. The following are equivalent.

(1) $\mathfrak{R}^{\circ}$ is o-minimal and $\mathfrak{R} \vDash \mathrm{NDG}$.

(2) $\mathfrak{R}^{\circ}$ is o-minimal and the graph of every unary definable function is nowhere dense.

(3) For every $S \subseteq R$ and finite collection $\mathcal{A}$ of $S$-definable subsets of $R^{m+n}$ there is a decomposition $\mathcal{C}$ of $R^{m+n}$ by $S$-definable cells such that $\mathcal{C}_{x}$ is quasicompatible with $\mathcal{A}_{x}$ for every $x \in R^{m}$. 
(4) For every $A \subseteq R^{n}$ and finite collection $\mathcal{F}$ of definable functions $A \rightarrow R$ there is a decomposition $\mathcal{C}$ of $R^{n}$ such that for each $f \in \mathcal{F}$ and $C \in \mathcal{C}$ exactly one of the following holds:

(a) $A \cap C=\emptyset$.

(b) $C \subseteq A$ and $f\lceil C$ is continuous.

(c) $A$ is dense and co-dense in $C$, and $f$ is given piecewise by continuous definable functions $C \rightarrow R$ having pairwise disjoint graphs.

Proof. We show that $(3) \Rightarrow(2) \Rightarrow(1) \Rightarrow(4) \Rightarrow(3)$.

$(3) \Rightarrow(2)$. Setting $m=0$, we have that $\mathfrak{R}^{\circ}$ is o-minimal by quasidecomposition. Let $f$ be a definable unary function. We show that $\operatorname{graph}(f)$ is nowhere dense. Suppose to the contrary that graph $(f)$ is dense in some box $B$. Let $\mathcal{C}$ be a decomposition of $R^{2}$ such that $\mathcal{C}_{x}$ is quasicompatible with $\left\{B_{x}, \operatorname{graph}(f)_{x}\right\}$ for every $x \in R$. As $B$ is open, there is an open cell $C \in \mathcal{C}$ contained in $B$. As $\operatorname{graph}(f)$ is dense in $C$, there exists $x \in \pi C$ such that $(x, f(x)) \in C$. But $C_{x}$ is an open interval containing $\{f(x)\}=\{\operatorname{graph}(f)\}_{x}$, contradicting that $\mathcal{C}_{x}$ is quasicompatible with $\{\operatorname{graph}(f)\}_{x}$.

$(2) \Rightarrow(1)$. We proceed by induction on $n \geq 1$ to show that $\operatorname{graph}(f)$ is nowhere dense for every definable $f: R^{n} \rightarrow R$. The case $n=1$ holds by assumption, so let $n>1$ and assume the result for all positive integers less than $n$. Let $B \times(a, b) \times$ $(c, d) \subseteq R^{n-1} \times R \times R$ be a box. We must find a box disjoint from $\operatorname{graph}(f)$ and contained in $B \times(a, b) \times(c, d)$. For $x \in R^{n-1}$, let $f_{x}$ denote the unary function $t \mapsto f(x, t)$. Note that since graph $\left(f_{x}\lceil(a, b))\right.$ is nowhere dense and $\mathfrak{R}^{\circ}$ is o-minimal, the closure of $\operatorname{graph}\left(f_{x} \uparrow(a, b)\right)$ is a finite disjoint union of nonopen cells, none of which are vertical line segments. Define $g: B \times(a, b) \rightarrow R$ by

$$
g(x, t)= \begin{cases}\min \left((c, \infty) \cap \operatorname{cl}\left(\operatorname{graph}\left(f_{x}\right)\right)_{t}\right) & \text { if }(c, \infty) \cap \operatorname{cl}\left(\operatorname{graph}\left(f_{x}\right)\right)_{t} \neq \emptyset, \\ c & \text { otherwise, }\end{cases}
$$

and let $g_{x}$ denote the function $t \mapsto g(x, t):(a, b) \rightarrow R$. Then for each $x \in B$, there exists $s \in(a, b)$ such that $g_{x}\lceil(a, s)$ is either constant or strictly monotone. Consider the definable function $B \rightarrow R$ given by

$$
x \mapsto \sup \left\{s \in(a, b): g_{x}\lceil(a, s) \text { is either constant or strictly monotone }\} .\right.
$$

Inductively, the closure of its graph is a finite disjoint union of nonopen cells. Hence, by shrinking $B \times(a, b)$, we reduce to the case that for each $x \in B$ we have either $\inf g_{x}>c$ or $g_{x}=c$. Now, $x \mapsto \inf g_{x}: B \rightarrow R$ is definable, so inductively there exist a box $B^{\prime} \subseteq B, b^{\prime} \in(a, b)$, and $d^{\prime} \in(c, d)$ such that for all $(x, t) \in B^{\prime} \times\left(a, b^{\prime}\right)$ we have either $g(x, t)=c$ or $g(x, t) \geq d^{\prime}$. Then $B^{\prime} \times\left(a, b^{\prime}\right) \times\left(c, d^{\prime}\right)$ is disjoint from $\operatorname{graph}(f)$.

$(1) \Rightarrow(4)$ is a routine consequence of quasidecomposition, 4.15 and 1.20

$(4) \Rightarrow(3)$. First, observe that by passing to characteristic functions and applying quasidecomposition, $\mathfrak{R}^{\circ}$ is o-minimal. Moreover, $\mathfrak{R} \vDash N D G$, since the graph of each definable function is contained in the union of the graphs of finitely many continuous (possibly partial) functions, and graphs of continuous functions are nowhere dense. Hence, by 4.15, we also have $\mathfrak{R} \vDash$ UF. The rest of the proof is quite similar to that of cell decomposition in the o-minimal setting. We provide only an outline.

Define the q-cells in $R^{n}$ inductively by: (i) $R^{0}$ is the only q-cell in $R^{0}$; (ii) $Q \subseteq$ $R^{n+1}$ is a q-cell if and only if $Q$ is definable, the projection $\pi Q$ of $Q$ onto the first $n$ coordinates is a q-cell in $R^{n}$, and there is a cell $C \subseteq R^{n+1}$ such that either 
$Q=C \cap(\pi Q \times R)$ or $Q_{x}$ is dense and co-dense in $C_{x}$ for every $x \in \pi Q$. Next, define a q-decomposition of $R^{n}$ by replacing "cell" with "q-cell" in the definition of decomposition of $R^{n}$ ([10, Chapter 3]). A routine induction on $m$ shows that if $\mathcal{A}$ is a collection of subsets of $R^{m+n}$ and $\mathcal{B}$ is a q-decomposition of $R^{m+n}$ compatible with $\mathcal{A}$, then for every $x \in R^{m}, \mathcal{B}_{x}$ is a q-decomposition of $R^{n}$ compatible with $\mathcal{A}_{x}$. Hence, the proof is now reduced to showing that for every finite collection $\mathcal{A}$ of definable subsets of $R^{n}$ there is a q-decomposition $\mathcal{B}$ of $R^{n}$ compatible with $\mathcal{A}$. We proceed by induction on $n$. The case $n=0$ is trivial. Assume the result for a certain $n \geq 0$. Let $\mathcal{A}$ be a finite collection of definable subsets of $R^{n+1}$. Put

$$
Y=\bigcup_{A \in \mathcal{A}}\left\{(x, r) \in R^{n+1}: r \in \operatorname{bd}\left(\operatorname{cl}\left(A_{x}\right)\right) \cup \operatorname{bd}\left(\operatorname{int}\left(A_{x}\right)\right)\right\} .
$$

By o-minimality of $\mathfrak{R}^{\circ}$, each $Y_{x}$ is finite. By UF, there exists $N \in \mathbb{N}$ such that card $Y_{x} \leq N$ for each $x \in R^{n}$. The rest of the proof is a routine modification of [10, p. 55].

As a corollary of the preceding two results (compare with Theorem B),

4.17. Let $T \supseteq \mathrm{DLO}$ be complete and have a model $\mathfrak{M}$ such that $\mathfrak{M}^{\circ}$ is o-minimal and the graph of every unary function definable in $\mathfrak{M}$ is nowhere dense. Then $T$ satisfies all of $\mathrm{DC}, \mathrm{UF}, \mathrm{EP}$, and $\mathrm{NDG}$, and $\mathrm{Th}\left(\mathfrak{M}_{\emptyset}^{\circ}\right)$ is an open core of $T$.

This concludes Section 4

We have arrived at a turning point. For the remainder of the paper, we focus on examples (Sections 5 and 6), counterexamples (Section 7) and open issues (Section 8). In [28, two natural classes, dense (elementary) pairs and expansions by generic predicates, are given of expansions of the real field that are not o-minimal yet have o-minimal open core. A key difference is that NDG fails for the former and holds for the latter. In the next two sections, we show that these examples extend to our more general setting, and we investigate them in more detail.

\section{DenSe PAIRS}

In this section, we consider our first examples of non-o-minimal structures and theories having o-minimal open core. Given $T \supseteq$ DLO, a dense pair of models of $T$ is a structure $(\mathfrak{B}, A)$ such that $\mathfrak{A} \supsetneqq \mathfrak{B} \vDash T$ and $A$ is dense in $B$. The study of dense pairs can be traced back to Robinson [39] and Macintyre [23]. A basic example is $(\mathbb{R},<, \mathbb{Q})$, and it easy to see that DLO is an open core of $\operatorname{Th}(\mathbb{R},<, \mathbb{Q})$. We are interested here only in dense pairs of o-minimal expansions of ordered groups.

Throughout this section, let DOG $\subseteq T$ be o-minimal and complete in a language $L$. Let $U \notin L$ be a unary relation symbol, put $L^{\mathrm{d}}=L \cup\{U\}$, and let $T^{\mathrm{d}}$ be the $L^{\mathrm{d}}$-theory of dense pairs of models of $T$. The seminal work in this setting is due to van den Dries [9], where it is shown that $T^{\mathrm{d}} \vDash \mathrm{DC}+\mathrm{UF}$ and is complete. Moreover, by [9, Corollary 4.6] and 4.14, $T$ is an open core of $T^{\mathrm{d}}$. It can be shown that $T^{\mathrm{d}} \vDash$ NIP 2 But a number of good properties of $T$ are lost in passing to $T^{\mathrm{d}}$. We show below that $T^{\mathrm{d}}$ has neither atomic models, definable Skolem functions, elimination of imaginaries, nor, in most cases of interest, EP (hence, by 4.15, not NDG). Let $(\mathfrak{B}, A)$ denote an arbitrary model of $T^{\mathrm{d}}$.

$5.1\left(\right.$ [9, Lemma 3.2]). For every $S \subseteq A, \operatorname{dcl}_{(\mathfrak{B}, A)}(S)=\operatorname{dcl}_{\mathfrak{A}}(S)$.

\footnotetext{
${ }^{2} \mathrm{~A}$ paper is in preparation by Berenstein, Dolich, and Onshuus.
} 
Hence, by 4.7

\section{2. $T^{\mathrm{d}}$ has no atomic models.}

We do not yet know if it is impossible for $T^{\mathrm{d}}$ to have a prime model, but there are interesting cases where we do have answers. Of course, there is the usual consequence that $T^{\mathrm{d}}$ has no prime model if $L$ is countable, but also if $T$ has a model $\mathfrak{R}$ such that $\operatorname{dcl}_{\mathfrak{R}}(\emptyset)$ is dense in two distinct proper elementary extensions. (Recall that $T$ has definable Skolem functions, so $\operatorname{dcl}_{\mathfrak{R}}(\emptyset)$ is a prime model of $T$.)

5.3. A set is $\boldsymbol{A}$-small if it is definable in $(\mathfrak{B}, A)$ and contained in $f\left(A^{n}\right)$ for some $f: B^{n} \rightarrow B$ definable in $\mathfrak{B}$. It is immediate that images of $A$-small sets under unary functions definable in $\mathfrak{B}$ are again $A$-small, and that finite unions of $A$-small sets are $A$-small [9, Remark 1.4]. More difficult are that $A$-small sets have no interior [9, Lemma 4.1] and each unary function definable in $(\mathfrak{B}, A)$ agrees off an $A$-small set with a unary function definable in $\mathfrak{B}[9$, Theorem $3(1)]$.

We now show that $T^{\mathrm{d}}$ does not have definable Skolem functions. While this is an easy consequence of either 5.1 or 5.2 , we prefer the following more explicit result.

5.4. If $f: B \rightarrow A$ is definable in $(\mathfrak{B}, A)$, then there is an $A$-small set $S$ such that $f(B \backslash S)$ is finite. In particular, there is no unary function definable in $(\mathfrak{B}, A)$ such that $f(t) \in A \cap(t, \infty)$ for all sufficiently large $t \in B$.

Proof. Let $f: B \rightarrow A$ be definable in $(\mathfrak{B}, A)$. By 5.3 , there exist $g: B \rightarrow B$ definable in $\mathfrak{B}$ and an $A$-small set $S$ such that $g \uparrow(B \backslash S)=f \uparrow(B \backslash S)$. Then

$$
g(B)=g(S) \cup g(B \backslash S)=g(S) \cup f(B \backslash S) \subseteq g(S) \cup A,
$$

so $g(B)$ is $A$-small, and thus has no interior (again by [5.3). As $g(B)$ is definable in the o-minimal structure $\mathfrak{B}$, we have $g(B)$ finite, so $f(B \backslash S)$ is finite.

Next, $T^{\mathrm{d}}$ does not eliminate imaginaries.

5.5. There is no $f: B \rightarrow B^{n}$ definable in $(\mathfrak{B}, A)$ such that $x-y \in A \Leftrightarrow f(x)=f(y)$ for all $x, y \in B$.

Proof. Suppose otherwise. For each $i=1, \ldots, n$ there is an $A$-small set $S_{i}$ and $g_{i}: B \rightarrow B$ definable in $\mathfrak{B}$ such that $f_{i} \uparrow\left(B \backslash S_{i}\right)=g_{i} \uparrow\left(B \backslash S_{i}\right)$. Let $S$ be the union of the $S_{i}$ and put $g=\left(g_{1}, \ldots, g_{n}\right)$. Observe that $S$ is $A$-small and $g \uparrow(B \backslash S)=f \uparrow(B \backslash S)$. Fix some $0<c \in B \backslash A$. The translates $c+S$ and $1+S$ are $A$-small; hence so is the union of $S, c+S$, and $1+S$. As $A$-small sets have no interior, for every $b \in B$ there exists $t$ such that $t, c+t, 1+t \in(b, \infty) \backslash S$; then $g(t) \neq g(c+t)$ and $g(t)=g(1+t)$. But this is impossible: by the Monotonicity Theorem applied to each $g_{i}$, there exists $b \in B$ such that $g \uparrow(b, \infty)$ is either constant or injective.

5.6. The preceding result was inspired by a noteworthy classical setting. Suppose that $T$ has an Archimedean model and $L$ is countable. Then $T^{\mathrm{d}}$ has a model $(\mathfrak{B}, A)$ where $B=\mathbb{R}$ (recall $1.13(4)$ ) and $A$ is countable. Modulo Borel equivalence, the relation $x \sim y \Leftrightarrow x-y \in A$ is the Vitali relation (that is, with $A=\mathbb{Q}$ ), so there is no Borel map $f: \mathbb{R} \rightarrow \mathbb{R}^{n}$ such that $x-y \in A \Leftrightarrow f(x)=f(y)$ for all $x, y \in \mathbb{R}$. By [9, Theorem 1], every set definable in $(\mathfrak{B}, A)$ is Borel (indeed, a Boolean combination of $\mathrm{F}_{\sigma}$ sets).

We now consider whether EP and NDG are preserved. In most cases of interest, they are not, but there do exist some natural examples where they are. 
5.7. If $(\mathfrak{B}, A)$ has a pole, then $T^{\mathrm{d}} \not \models \mathrm{EP}$ and $T^{\mathrm{d}} \not \models \mathrm{NDG}$.

Note. The expression $T^{\mathrm{d} \not \models \mathrm{EP}}$ does not imply that $(\mathfrak{B}, A) \not \models \mathrm{EP}$. For example, if $\mathfrak{B}$ is prime over $\mathfrak{A}$ and one additional element, then $\operatorname{dcl}$ in $(\mathfrak{B}, A)$ is the same as in $\mathfrak{B}$ by 5.1

Proof. Let $I$ be a bounded open interval and $f: I \rightarrow B$ be injective and definable in $(\mathfrak{B}, A)$ such that $f(I)$ is an unbounded interval. Now, $f$ agrees off an $A$-small set with a function $I \rightarrow R$ definable in $\mathfrak{B}$, so by the Monotonicity Theorem, $\mathfrak{B}$ has a pole. By 1.13, we reduce to the case that $T$ extends the theory of ordered fields.

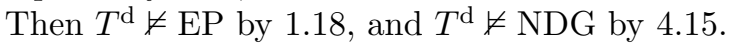

The assumption of a pole is not necessary, but the result is cumbersome to state otherwise. The key point of the proof is that $(\mathfrak{B}, A) \emptyset$-defines an ordered ring on some interval. By the Trichotomy Theorem [32, all that is needed for this is a suitably nontrivial definable curve. For example, if $T$ is the theory of the expansion of $(\mathbb{R},<,+, 1)$ by the set $\left\{\left(t, t^{2}\right): 0<t<1\right\}$, then $T^{\mathrm{d}} \not \models \mathrm{EP}$.

Remark. It is easy to prove directly that if $(K,<,+, \cdot)$ is an ordered field and $J$ is a dense proper subfield, then $(K,<,+, \cdot, J)$ defines a unary function whose graph is dense. See [9, pp. 62-63] or [28, p. 206].

On the other hand, it is possible that $T^{\mathrm{d}} \vDash \mathrm{EP}+\mathrm{NDG}$, as we now show. Fix an ordered field with underlying set $K$, and let $T_{K}$ be the theory of $K$ as an ordered vector space, that is,

$$
T_{K}:=\operatorname{Th}\left(K,<,+,-, 0,1,(t \mapsto \alpha t)_{\alpha \in K}\right) .
$$

It is well known (or easy to check) that $T_{K}$ admits QE, see [10, Chapter $1, \S 7$ ] for a proof, and thus is o-minimal, so the theory $T_{K}^{\mathrm{d}}$ of its dense pairs exists.

5.8. $T_{K}^{\mathrm{d}}$ admits $Q E$ and $\mathrm{dcl}$ in $T_{K}^{\mathrm{d}}$ is the same as in $T_{K}$.

Proof. QE can be obtained via explicit elimination of single existential quantifiers ranging over conjunctions of atomic and negated atomic formulas. We leave the details as an exercise. We now establish the preservation of dcl. Let $(\mathfrak{B}, A) \vDash T_{K}^{\mathrm{d}}$ and $C \subseteq B^{m+1}$ be $\emptyset$-definable. It suffices to exhibit $D \subseteq B^{m+1}$ that is $\emptyset$-definable in $\mathfrak{B}$ such that for every $x \in B^{m}$, if $C_{x}$ is finite, then so is $D_{x}$, and $C_{x} \subseteq D_{x}$. By $\mathrm{QE}$, we reduce to the case that

$C=D \cap \bigcap_{i=1}^{M}\left\{(x, t) \in B^{m+1}: f_{i}(x)+t \in A\right\} \cap \bigcap_{j=1}^{N}\left\{(x, t) \in B^{m+1}: g_{j}(x)+t \notin A\right\}$,

where $D$ is $\emptyset$-definable in $\mathfrak{B}$ and the $f_{i}, g_{j}$ are affine $K$-linear. Fix $x \in B^{m}$ such that $C_{x}$ is finite and nonempty. We show that $D_{x}$ is finite. By o-minimality of $\mathfrak{B}$, it suffices to show that $D_{x}$ has no interior. This follows from observing that if $G_{1}, \ldots, G_{M}, H_{1}, \ldots, H_{N} \subseteq B$ are cosets of $A$, then the set $\bigcap_{i=1}^{M} G_{i} \cap \bigcap_{j=1}^{N}\left(B \backslash H_{j}\right)$ is either empty or dense in $B$.

Hence, by 1.20 ,

5.9. $T_{K}^{\mathrm{d}} \vDash \mathrm{EP}+\mathrm{NDG}$.

Remark. It is known (or easy to check) that dcl in $T_{K}$ is just $K$-linear spanning, so the same is true in $T_{K}^{\mathrm{d}}$. 
Related examples. Some interesting theories related to dense pairs have recently come to light via work of others [1,2,41. We shall not attempt to describe them fully, but as examples, if $E$ is either a noncyclic, finitely generated subgroup of $\left(\mathbb{R}^{>0}, \cdot\right)$, or the set of all complex roots of unity (regarded as a subset of $\mathbb{R}^{2}$ ), then the theory of real closed fields is an open core of $\operatorname{Th}(\mathbb{R},+, \cdot, E)$. In both cases, it suffices by 4.14 to show that in every $\mathfrak{M} \equiv(\mathbb{R},+, \cdot, E)$, every open unary definable set is a finite union of open intervals and every cofinitely continuous unary definable function is given piecewise by $M$-semialgebraic functions. The condition on open sets is shown more or less explicitly in [2]. The condition on functions is not, but this follows from results analogous to [9, Theorem 3.1]. Moreover, there are results similar to [9, Theorem 1] yielding that every set definable in $(\mathbb{R},+, \cdot, E)$ is Borel, so easy modifications of 5.6 show that $\operatorname{Th}(\mathbb{R},+, \cdot, E)$ does not eliminate imaginaries. By 1.18 and 4.15. EP and NDG fail as well. We imagine that modifications of our arguments would also yield failure of definable Skolem functions and nonexistence of atomic (hence also prime) models.

\section{Generic predichtes}

We now consider a second class of theories that are not o-minimal but have o-minimal open cores. As usual, $T$ denotes a theory in a language $L$. Let $L^{\mathrm{g}}$ be an extension of $L$ by a new unary relation symbol. Suppose that $T$, regarded as an $L^{\mathrm{g}}$-theory, has a model companion $T^{\mathrm{g}}$. Then any $L^{\mathrm{g}}$-extension of $T^{\mathrm{g}}$ is called an extension of $T$ by a generic predicate. Given a structure $\mathfrak{M}$ and $G \subseteq M$, we say that $G$ is generic for $\mathfrak{M}$ if $(\operatorname{Th}(\mathfrak{M}))^{\mathrm{g}}$ exists and has $(\mathfrak{M}, G)$ as a model. Seminal work is due to Chatzidakis and Pillay [3, §2], who we cite often. For convenience in applying their results, we assume throughout that $T$ is complete and admits QE as an $L$-theory, a global assumption in [3. By [3, 2.4, 2.11], $T^{\mathrm{g}}$ exists if and only if $T \vDash$ UF. Hence, if every model of $T$ has o-minimal open core, then $T^{\mathrm{g}}$ exists by 4.2 We use these facts in the sequel without mention. We are interested here mainly in the case that $T$ is o-minimal, but we work in greater generality when it is easy to do so. We now assume moreover that $T \supseteq$ DLO and our structure $\mathfrak{R}$, which may or may not be a model of $T$, admits $\mathrm{QE}$ in its given language. We are ready to state our first result.

6.1. If $T$ is o-minimal, then $(\mathfrak{R}, G)^{\circ}={ }_{\mathrm{df}} \mathfrak{R}$ for every $(\mathfrak{R}, G) \vDash T^{\mathrm{g}}$.

It is immediate from [3, 2.4] (see 6.8 below for details) that if $G$ is generic for $\mathfrak{R}$, then $G$ is dense and co-dense in $R$. Thus, we have another supply of non-o-minimal theories that have complete o-minimal open cores. We analyze the situation as we did for dense pairs. In order to smooth the exposition, we defer the proof of 6.1 . as well as most of the proofs in this section, until after all results are stated and discussed.

Before proceeding, we give some history. For use in [28, Miller desired an example, as concrete as possible, of a polynomially bounded expansion of the real field that is not o-minimal. (By results in 28, such a structure has o-minimal open core and satisfies NDG for the unary definable functions.) In response, Harvey Friedman produced examples such that every definable set is Borel, indeed, a Boolean combination of $\mathrm{F}_{\sigma}$ sets. In doing so, he developed a general method for producing non-o-minimal expansions $\widehat{\Re}$ of o-minimal $\mathfrak{R}$ such that every unary function definable in $\widehat{\Re}$ is given piecewise by functions definable in $\mathfrak{R}$; see [16]. Remarkably, 
this method turns out to be the same as genericity over o-minimal expansions of densely ordered groups (see Fratarcangeli [15]) though the notions were developed independently, almost simultaneously, and for very different reasons. With minor and predictable modification (see 6.9 below), the notions coincide for all o-minimal structures. (Friedman's construction reflects that, for the purposes of 28, information was needed only on the unary functions definable in fixed structures.) Fratarcangeli used this connection to show that if $T$ is o-minimal, then the elimination of imaginaries is preserved and versions of some of our results here hold; in particular, a proof of 6.1 was given. Nevertheless, none of our results here depend on [15, 16, 28 and our proof of 6.1 differs enough to warrant inclusion here, though it is fair to say that our 6.4 below is motivated as much by $[16,28$ as by $[3$.

Actually, 6.1 holds in greater generality: It suffices to assume only that every model $T$ has o-minimal open core. As we shall see in our proof of 6.1, this generalization is very easy if moreover $T \vDash N D G$. But without this or other extra assumptions such as group structure, the proof is longer and more model-theoretically technical than we would like for this paper. Hence, we shall prove this generalization elsewhere (a paper is in preparation) as part of a more extensive investigation into results associated with passing from $T$ to $T^{\mathrm{g}}$.

We begin our analysis. Crucial for further developments is that dcl in $T^{\mathrm{g}}$ is the same as in $T$ [3, 2.6.3] (so we dispense with subscripting dcl in this section). Hence, by $1.17,1.20$, and 6.1 .

6.2. (1) If $\mathfrak{R} \vDash \mathrm{UF}$ and $G$ is generic for $\mathfrak{R}$, then every function definable in $(\mathfrak{R}, G)$ is given piecewise by functions definable in $\mathfrak{R}$ (using the same parameters).

(2) If $T \vDash \mathrm{EP}$, then $T^{\mathrm{g}}$ exists and $T^{\mathrm{g}} \vDash \mathrm{UF}+\mathrm{EP}$.

(3) If $T \vDash \mathrm{UF}+\mathrm{NDG}$, then $T^{\mathrm{g}} \vDash \mathrm{NDG}$.

(4) If $T$ is o-minimal, then $T^{\mathrm{g}} \vDash \mathrm{UF}+\mathrm{EP}+\mathrm{NDG}$ and has $T$ as an open core.

Remark. According to [3, 2.12.4], UF is always preserved in passing from $T$ to $T^{\mathrm{g}}$, but there is a flaw in the suggested method of proof. (It is not true in general that preservation of acl implies preservation of UF.) Chatzidakis and Pillay have acknowledged the oversight and outlined an alternate approach.

In contrast, some good properties of $T$ fail for $T^{\mathrm{g}}$. As we have already noted, every model of $T^{\mathrm{g}}$ defines unary sets that are dense and co-dense, so constructibility of definable sets fails. By [3, 2.10], NIP fails except in trivial cases. By [3, 2.6.1], completeness usually fails as well, because each $(\Re, G) \vDash T^{\mathrm{g}}$ is axiomatized over $T^{\mathrm{g}}$ by expressing which elements of $\operatorname{dcl}(\emptyset)$ are contained in $G$. We show that

\subsection{No completion of $T^{\mathrm{g}}$ has definable Skolem functions.}

Existence of prescribed models. Given a model of $T \vDash U F$, does it expand to a model of $T^{\mathrm{g}}$ ? Equivalently, if $\mathfrak{R} \vDash \mathrm{UF}$, do there exist generic sets for $\mathfrak{R}$ ? We do not know the answer in this generality, but we do have some good sufficiency results in the o-minimal setting, especially over $\mathbb{R}$.

6.4. Suppose that $\operatorname{card} I=\operatorname{card} R$ for every interval $I \subseteq R$.

(1) If there are at most ( $\operatorname{card} R$ )-many continuous functions $B \rightarrow R$ as $B$ ranges over all boxes, then there are subsets of $R$ that are generic for every o-minimal expansion of $(R,<)$. 
(2) If $\Re$ is o-minimal and card $R \geq \operatorname{card} L$, then there exist subsets of $R$ that are generic for $\mathfrak{R}$.

As immediate consequences,

6.5. (1) There exist subsets of $\mathbb{R}$ that are generic for every o-minimal expansion of $(\mathbb{R},<)$.

(2) If $T$ is o-minimal and extends the theory of ordered fields, then every model of $T$ having cardinality at least that of $L$ expands to a model of $T^{\mathrm{g}}$.

(3) If $T$ is countable, o-minimal, and extends the theory of ordered fields, then every model of $T$ expands to a model of $T^{\mathrm{g}}$.

Atomic models. We now touch on the issue of atomic models by considering the o-minimal field case.

6.6. Suppose that $T$ is o-minimal and extends the theory of ordered fields.

(1) A model $(\mathfrak{R}, G)$ of $T^{\mathrm{g}}$ is atomic if and only if $R=\operatorname{dcl}(\emptyset)$.

(2) There exist $\mathfrak{M} \vDash T^{\mathrm{g}}$ such that $\mathrm{Th}(\mathfrak{M})$ has no atomic models.

(3) If card $L \leq \operatorname{card}(\operatorname{dcl}(\emptyset))$, then $T^{\mathrm{g}}$ has an atomic model.

(4) If $T$ is countable, then there exist $\mathfrak{A}, \mathfrak{B} \vDash T^{\mathfrak{g}}$ such that $\operatorname{Th}(\mathfrak{A})$ has a prime model and $\operatorname{Th}(\mathfrak{B})$ does not.

Proof. Item (1) is immediate from 6.1, 4.7, and preservation of dcl. Item (4) is immediate from (2), (3), and classical model theory.

(2). It follows from the axiomatization of $T^{\mathrm{g}}[3,2.4]$ that there exist $(\Re, G) \vDash T^{\mathrm{g}}$ with $\operatorname{dcl}(\emptyset) \cap G=\emptyset$. By $(1), \operatorname{Th}(\Re, G)$ has no atomic models.

(3). By definability of Skolem functions, $T$ has a model $\mathfrak{R}$ such that $R=\operatorname{dcl}(\emptyset)$. By 6.5. $\mathfrak{R}$ expands to a model of $T^{\mathrm{g}}$, which is atomic by (1).

Proofs. We now work toward the proofs of 6.1, 6.3, and 6.4, but we do them in reverse order.

Given $n \in \mathbb{N}, G \subseteq R$, and $J \subseteq\{1, \ldots, n\}$, put $G^{J}=\left\{y \in R^{n}: y_{j} \in G \Leftrightarrow j \in\right.$ $J\}$. The notation suppresses the dependence on $n$, but the context should resolve ambiguity.

We begin with a restatement of [3, 2.4] for saturated structures.

6.7. If $\mathfrak{R} \vDash \mathrm{UF}$ and is saturated, then the following are equivalent.

(1) $G$ is generic for $\mathfrak{R}$.

(2) For every finite $S \subseteq R$ and $A \subseteq R^{n}$ that is $S$-definable in $\mathfrak{R}$, if there is a point in $A$ having pairwise distinct coordinates, none of which belong to $\operatorname{dcl}(S)$, then $A \cap G^{J} \neq \emptyset$ for every $J \subseteq\{1, \ldots, n\}$.

6.8. It follows that $G$ is dense and co-dense in $R$ for every saturated $(\Re, G) \vDash T^{\mathrm{g}}$, hence also in every model of $T^{\mathrm{g}}$.

In the o-minimal setting, genericity can be expressed concretely without having to assume saturation.

6.9. If $\mathfrak{R}$ is o-minimal, then the following are equivalent.

(1) $G$ is generic for $\mathfrak{R}$.

(2) For every continuous $f=\left(f_{1}, \ldots, f_{n}\right): B \rightarrow R^{n}$ definable in $\mathfrak{R}$ such that $B$ is a box, every point in $f(B)$ has pairwise distinct coordinates, and every $f_{j}$ is strictly monotone in each coordinate, and for every $J \subseteq\{1, \ldots, n\}$, we have $f^{-1}\left(G^{J}\right) \neq \emptyset$. 
Remark. By [15, this holds with "interval" in place of "box" if moreover $\Re$ expands a densely ordered group.

Proof. As the conditions of (2) are first order, it suffices to consider the case that $\mathfrak{R}$ is saturated. We now show that (2) is equivalent to $6.7(2)$.

Assume that 6.7 (2) holds. We must show that $f^{-1}\left(G^{J}\right) \neq \emptyset$ with all data as in (2). Let $S$ be the parameters used in defining $f$. By saturation, there exists $x \in B$ independent over $S$. An easy induction on the dimension of $B$ yields that no coordinate of $f(x)$ is defined over $S$. By applying $6.7(2)$ to $f(B)$, there exists $y \in f(B) \cap G^{J}$, that is, $f^{-1}\left(G^{J}\right) \neq \emptyset$.

Assume that (2) holds. Let $S \subseteq R$ be finite and $A \subseteq R^{n}$ be $S$-definable in $\Re$. Suppose that $A$ contains a point having pairwise distinct coordinates, none of which are defined over $S$. Note that $A$ is infinite. Let $J \subseteq\{1, \ldots, n\}$. We must show that $A \cap G^{J} \neq \emptyset$. By considering the identity function on $R^{n}$, we see that $G^{J}$ is dense in $R^{n}$, so we may reduce to the case that $A$ has no interior. Hence, we may reduce to the case that $A$ is an infinite nonopen cell, all of whose points have pairwise distinct coordinates. Put $m=\operatorname{dim} A$. After permutation of coordinates, it follows from "regular" cell decomposition [10, p. 58] that $A$ contains the graph of a continuous map $g: B \rightarrow R^{n-m}$ definable in $\mathfrak{R}$, where $B$ is a box in $R^{m}$ and each $g_{j}$ is strictly monotone in each coordinate. Define $f: B \rightarrow R^{n}$ by $f(x)=(x, g(x))$. Observe that $f(B)=\operatorname{graph}(g) \subseteq A$ and $f$ satisfies the hypotheses of (2). Hence, $A \cap G^{J} \neq \emptyset$.

Now, 6.4 is immediate from 6.9 and the following observation, whose proof is a routine transfinite induction that we leave to the reader.

6.10. Suppose that $\operatorname{card} I=\operatorname{card} R$ for every interval $I \subseteq R$. Let $\mathcal{F}$ be a collection of maps of the form $f=\left(f_{1}, \ldots, f_{n}\right): B \rightarrow R^{n}$, where $B \subseteq R^{m}$ is a box, every point in $f(B)$ has pairwise distinct coordinates, and each $f_{j}$ is strictly monotone in each coordinate. (Both $m$ and $n$ are allowed to vary.) If card $\mathcal{F} \leq \operatorname{card} R$, then there exists $G \subseteq R$ such that $f^{-1}\left(G^{J}\right) \neq \emptyset$ for every $f \in \mathcal{F}$ and $J \subseteq\{1, \ldots, n\}$.

Proof of 6.3. We show that no completion of $T^{\mathrm{g}}$ has definable Skolem functions; indeed, no model $(\Re, G)$ of $T^{\mathrm{g}}$ defines a unary function $g: R \rightarrow G$ such that $g(t)>t$ for all sufficiently large $t$ (compare with [5.4). It suffices to consider the case that $(\mathfrak{R}, G)$ is saturated. Let $c \in R$. We show that $g(t) \leq t$ for some $t>c$. Suppose otherwise. By 6.2, $g$ is given piecewise by functions $f_{1}, \ldots, f_{n}$ definable in $\mathfrak{R}$. By replacing each $f_{i} \uparrow(c, \infty)$ with $t \mapsto f_{j}(t)$, where $j$ is minimal such that $f_{j}(t)>t$, we reduce to the case that $f_{1}(t), \ldots, f_{n}(t)>t$ for all $t>c$. After re-indexing, there exists $m \in\{1, \ldots, n\}$ such that the set

$$
A:=\left\{t>c: f_{1}(t), \ldots, f_{m}(t) \text { are pairwise distinct and } f_{m}(t)=\cdots=f_{n}(t)\right\}
$$

is unbounded above. Let $S$ be the parameters used in defining $A$. By saturation, $\operatorname{dcl}(S)$ is bounded, so there exists $a \in A$ such that $a>\operatorname{dcl}(S)$. As $a<f_{1}(a), \ldots, f_{m}(a)$, it follows that no $f_{i}(a)$ is defined over $S$. By applying 6.7 to the set $\left\{\left(f_{1}(t), \ldots, f_{m}(t)\right): t \in A\right\}$, there exists $t>c$ such that $f_{1}(t), \ldots, f_{n}(t) \notin G$, contradicting that $g(t) \in G$.

For saturated models, the model completeness of $T^{\mathrm{g}}$ improves to a $\mathrm{QE}$ result. 
6.11. If $\mathfrak{R} \vDash \mathrm{UF}$ is saturated and $G$ is generic for $\mathfrak{R}$, then every subset of $R^{m}$ definable in $(\Re, G)$ is a finite union of sets of the form $f^{-1}\left(G^{J}\right)$, where $f: R^{m} \rightarrow R^{n}$ is definable in $\mathfrak{R}$ and $J \subseteq\{1, \ldots, n\}$.

Proof. Fix $a \in G$ and $b \in R \backslash G$. Identify sets definable in $\Re$ with characteristic functions relative to $\{a, b\}$. Then every set definable in $\mathfrak{R}$ is of the form $g^{-1}(G)$ for some $g$ definable in $\mathfrak{R}$. By [3, 2.6.4], saturation, and compactness, every set definable in $(\Re, G)$ is a Boolean combination of sets of the form $g^{-1}(G)$, where $g$ is definable in $\mathfrak{R}$. By passing to disjunctive normal form, every set definable in $(\Re, G)$ is a finite union of sets of the form $\bigcap_{j=1}^{n} f_{j}^{-1}\left(E_{j}\right)$, where $E_{j} \in\{G, R \backslash G\}$ and each $f_{j}$ is definable in $\mathfrak{R}$. Put $f=\left(f_{1}, \ldots, f_{n}\right)$ and $J=\left\{j: E_{j}=G\right\}$.

Proof of 6.1. We establish a somewhat stronger result. Suppose only that some model of $T$ has o-minimal open core and satisfies NDG. By 4.15, every model of $T$ has o-minimal open core and $T \vDash \mathrm{NDG}+\mathrm{UF}$. Let $(\mathfrak{R}, G) \vDash T^{\mathrm{g}}$. We show that $(\mathfrak{R}, G)^{\circ}=\mathfrak{R}^{\circ}$. By 4.11 and $6.2(1)$, it suffices to show that $(\mathfrak{R}, G) \vDash \mathrm{UF}$ and every unary closed set definable in $(\mathfrak{R}, G)$ is a finite union of points and closed intervals. We may assume that $(\Re, G)$ is saturated. It suffices now by [1.16 and 6.11 to show that if $f=\left(f_{1}, \ldots, f_{n}\right): R \rightarrow R^{n}$ is definable in $\mathfrak{R}$ and $J \subseteq\{1, \ldots, n\}$, then $\operatorname{cl}\left(f^{-1}\left(G^{J}\right)\right)$ is a finite union of points and closed intervals. By quasidecomposition, there is decomposition of $R^{2}$ quasicompatible with the graphs of the $f_{i}$. By NDG, each $\operatorname{cl}\left(\operatorname{graph}\left(f_{i}\right)\right)$ is a finite union of nonopen cells of the decomposition. By monotonicity, we may assume that every nonopen cell of the decomposition whose projection onto the first coordinate has interior is the graph of a function from an open interval $I$ into $R$ that is either constant or injective. Thus, after refining, we obtain a finite partition $\mathcal{A}$ of $R$ by sets definable in $\mathfrak{R}$ such that for every $A \in \mathcal{A}$ :

(1) $A$ is either a point or densely contained in an open interval;

(2) each $f_{i} \uparrow A$ is either constant or injective;

(3) for every $i$ and $j$, either $f_{i} \uparrow A=f_{j} \uparrow A$ or their graphs are disjoint.

We now fix $A \in \mathcal{A}$ that is not a point. After re-indexing, there exists $m \leq n$ such that for all $t \in A$, we have $f_{1}(t), \ldots, f_{m}(t)$ pairwise distinct and $f_{m}(t)=\cdots=f_{n}(t)$. As our goal now is to understand $\operatorname{cl}\left(A \cap f^{-1}\left(G^{J}\right)\right)$, it suffices to consider the case $m=n$; that is, we may assume that the graphs of the $f_{i} \uparrow A$ are pairwise disjoint. Those $f_{i}$ that are constant on $A$ give rise only to trivialities, so we assume that each $f_{i} \uparrow A$ is injective. It suffices now to show that $f^{-1}\left(G^{J}\right)$ is dense in $A$. Let $I$ be an interval that intersects $A$. Let $S$ be the set of parameters used in defining $f \uparrow(A \cap I)$. By saturation, we have $\operatorname{card}(A \cap I)=\operatorname{card} R>\operatorname{card}(\operatorname{dcl}(S))$, so there exists $t \in A \cap I \backslash \operatorname{dcl}(S)$. As each $f_{i} \uparrow A$ is injective, no $f_{i}(t)$ is defined over $S$. By applying 6.7 to the image $f(A \cap I)$, we see that $f^{-1}\left(G^{J}\right)$ intersects $A \cap I$, as was to be shown.

\section{Optimality}

We now consider the extent of optimality of some of the results of this paper via a collection of examples of structures of the form $(\mathfrak{M}, X) \vDash N D G$, where $\mathfrak{M}$ is o-minimal, $X \subseteq M$ is locally closed, and $(\mathfrak{M}, X)$ is not o-minimal. As the $X$ are locally closed, we have $(\mathfrak{M}, X)^{\circ}={ }_{\mathrm{df}}(\mathfrak{M}, X)$ by 1.4 and so $(\mathfrak{M}, X)^{\circ}$ is not o-minimal. All but one of the examples also satisfy UF, and all but two satisfy DC. 
Cantor sets. We show that the group structure is necessary in 2.14, hence also in Theorem A, even in the presence of NDG. Let $E \subseteq \mathbb{R}$ be a Cantor set; that is, $E$ is nonempty, compact, and has neither interior nor isolated points. Evidently, $(\mathbb{R},<, E)$ is not locally o-minimal, but more is true: As the set of left endpoints of the complementary intervals of $E$ is definable, $(\mathbb{R},<, E)$ defines nonempty sets that have no locally closed points (recall 2.10). Nevertheless, $(\mathbb{R},<, E) \vDash \mathrm{UF}+\mathrm{NDG}$. Indeed, more is true:

7.1. In every model of $\operatorname{Th}(\mathbb{R},<, E)$, every definable set either has interior or is nowhere dense, and is finite if discrete.

(By 1.16, UF then holds as well.)

Proof. First, for ease of notation only, we assume that $\min E=0$ and $\max E=$ 1. (Of course, this holds if $E$ is the classical middle-thirds Cantor set.) Define $\lambda, \rho: \mathbb{R} \rightarrow \mathbb{R}$ by $\lambda(t)=\rho(t)=0$ for $t \leq 0, \lambda(t)=\rho(t)=1$ for $t \geq 1$, and

$$
\begin{aligned}
& \lambda(t)=\sup (E \cap(-\infty, t)), \\
& \rho(t)=\inf (E \cap(t,+\infty))
\end{aligned}
$$

for $0<t<1$. Put $\mathfrak{R}=(\mathbb{R},<, \lambda, \rho, 0,1)$. We have $t \in[0,1] \backslash E$ if and only if $\lambda(t)<t<\rho(t)$, so $\mathfrak{R}=$ df $(\mathbb{R},<, E)$. Every term of $\mathfrak{R}$ is equivalent to one of $0,1, v$, $\lambda v$ or $\rho v$, for a variable $v$. It is an exercise to check that $\mathfrak{R}$ admits $\mathrm{QE}$ via explicit elimination of single existential quantifiers ranging over conjunctions of equations and strict inequalities of terms. The result now follows easily.

7.2. Observe that for each $n$ there are only finitely many $\emptyset$-definable $n$-ary sets, thus providing a more direct proof of UF, as well as showing that $\operatorname{Th}(\mathbb{R},<, E)$ is $\aleph_{0}$-categorical.

7.3. We show that "closed" is necessary in 2.4. Define $\mu: \mathbb{R} \rightarrow \mathbb{R}$ by $\mu(t)=$ $(\lambda(t)+\rho(t)) / 2$ if $\lambda(t)<\rho(t)$, and $\mu(t)=0$ otherwise. Then $X:=\{t \in \mathbb{R}: \mu(t) \neq 0\}$ is the set of midpoints of the bounded complementary intervals of $E$. Observe that $X$ is infinite and discrete, and $E=\operatorname{fr}(X)$. Put $\mathfrak{R}^{\prime}=(\mathfrak{R}, \mu)$, with $\mathfrak{R}$ as in the proof of 7.1. Again, $\mathfrak{R}^{\prime}$ admits $\mathrm{QE}$, and for each $n$ there are only finitely many $\emptyset$-definable $n$-ary sets. It follows that $(\mathbb{R},<, X) \vDash \mathrm{UF}$ and every definable set either has interior or is nowhere dense.

We include the next two examples to show that DC is not necessary for good behavior. The first (convex pairs) is weakly o-minimal but not o-minimal, while the second (tame pairs) is locally o-minimal but not weakly o-minimal.

Convex pairs. Let $T$ be a complete o-minimal theory extending the theory of ordered fields. Let $T^{\mathrm{c}}$ be the theory of all structures of the form $(\mathfrak{B}, V)$, where $\mathfrak{B} \vDash$ $T$ and $V \subsetneq B$ is convex and closed under all $\emptyset$-definable continuous functions. For example, if $\mathfrak{A} \supsetneqq \mathfrak{B}$ and $V$ is the convex hull of $A$ in $\mathfrak{B}$, then $(\mathfrak{B}, V) \vDash T^{\mathrm{c}}$. Observe that $(\mathfrak{B}, V) \not \models \mathrm{DC}$ and $V$ is $\mathrm{CBD}$ in $(\mathfrak{B}, V)$. By van den Dries and Lewenberg [12, $T^{\mathrm{c}}$ is complete and, relative to $T$, admits $\mathrm{QE}$ and is universally axiomatizable. It follows that $T^{\mathrm{c}}$ is weakly o-minimal, every set definable in any model of $T^{\mathrm{c}}$ is 
constructible, $\mathrm{dcl}_{T^{\mathrm{c}}}=\mathrm{dcl}_{T}$, and $T^{\mathrm{c}}$ has definable Skolem functions [8, 2.6]. By [24, 7.3], $T^{\mathrm{c}} \vDash$ NIP. In summary, all of the following are preserved in extending $T$ to $T^{\mathrm{c}}$ : completeness, weak o-minimality (hence also UF), constructibility of definable sets (hence also NDG), dcl (hence also EP), definability of Skolem functions, and NIP. See [4,8, 12,24, for more information and related examples.

Tame pairs. Let $T$ be a complete o-minimal theory extending the theory of ordered fields. Let $T^{\mathrm{t}}$ be the theory of all structures $(\mathfrak{B}, A)$, where $\mathfrak{A} \supsetneqq \mathfrak{B} \vDash T$ and for every $b$ in the convex hull of $A$ there is a unique $\phi(b) \in A$ such that $|b-\phi(b)|<\epsilon$ for all $\epsilon \in A^{>0}$. If $T$ is Archimedean, then $T^{\mathrm{t}}$ has a model $(\mathfrak{B}, A)$ with $A=\mathbb{R}$ and $\mathfrak{B}$ the Hardy field of $\mathfrak{A}$. Now, $A$ is $\mathrm{CBD}$ and discrete in $(\mathfrak{B}, A)$, but has no supremum, and $B \backslash A$ has infinitely many convex components. Hence, $(\mathfrak{B}, A) \not \models \mathrm{DC}$ and is not weakly o-minimal. By [12, $T^{\mathrm{t}}$ is complete. Extend $\phi$ to be defined on all of $B$ by setting $\phi(b)=0$ for $b$ not in the convex hull of $A$. Evidently, $\phi$ is locally constant, hence continuous, and $(\mathfrak{B}, A)$ is $\emptyset$-interdefinable with $(\mathfrak{B}, \phi)$. By $[12,(\mathfrak{B}, \phi)$ admits $\mathrm{QE}$ relative to $\mathfrak{B}$. Hence, every set definable in $(\mathfrak{B}, A)$ is constructible. At present, tame pairs are not as well documented as dense pairs and convex pairs, so we shall have to do a bit of work to establish that

\section{4. $(\mathfrak{B}, A) \vDash \mathrm{UF}$ and is locally o-minimal.}

As this is but a side issue for this paper, we give only a

Sketch of proof. By extending $T$ by all of its definable Skolem functions, we reduce to the case that $T$ admits $\mathrm{QE}$, its language $L$ has no relation symbols other than $<$, and every function $\emptyset$-definable in $\mathfrak{B}$ is given by a function symbol from $L$. Let $L^{\prime}$ be the extension of $L$ by a new unary function symbol for $\phi$, and regard $(\mathfrak{B}, \phi)$ as an $L^{\prime}$-structure. By [12, $(\mathfrak{B}, \phi)$ admits QE. As local o-minimality and UF are elementary, we may take $(\mathfrak{B}, \phi)$ to be as saturated as needed. Let $S \subseteq B$ be finite. By induction on complexity, for every finite set $\Sigma$ of unary $L_{S}^{\prime}$-terms there is a closed discrete $(A \cup S)$-definable $D \subseteq B$ such that for each convex component $C$ of $B \backslash D$ and $\sigma \in \Sigma$ there exists $g: B \rightarrow B,(A \cup S)$-definable in $\mathfrak{B}$, such that $\sigma\lceil C=g\lceil C$, which is either constant or continuous and strictly monotone, and $\phi \circ g\lceil C$ is constant. Hence, by $\mathrm{QE},(\mathfrak{B}, \phi)$ is locally o-minimal and every unary set $S$-definable in $(\mathfrak{B}, \phi)$ either has interior or is contained in $\operatorname{dcl}_{\mathfrak{B}}(A \cup S)$. By compactness, every unary set definable in $(\mathfrak{B}, \phi)$ then either has interior or is contained in some $f\left(A^{n}\right)$, where $f: B^{n} \rightarrow B$ is definable in $\mathfrak{B}$. Toward a contradiction, suppose that $(\mathfrak{B}, \phi) \not \models$ UF. By 1.16. $(\mathfrak{B}, \phi)$ defines an infinite unary $Y$ such that every infinite definable $X \subseteq Y$ contains infinitely many elements having successors in $X$. As $Y$ has no interior, it is contained in some $f\left(A^{n}\right)$, where $f: B^{n} \rightarrow B$ is definable in $\mathfrak{B}$. By arguing as in [9, 4.3], but using [11, 8.1] instead of [9, Theorem 2], $Y$ is a finite union of sets of the form $f\left(C \cap A^{n}\right)$, where $C$ is an open $C^{1}$-cell of $\mathfrak{B}$ and $f: C \rightarrow B$ is $C^{1}$ and definable in $\mathfrak{B}$. Routine modification of the proof of [28, Lemma, p. 206] yields that $Y$ contains a definably homeomorphic image $X$ of a set of the form $I \cap A$, where $I$ is an open interval of $B$ contained in the convex hull of $A$. But no element of $A$ has a successor, so the same is true of $X$, contradicting our earlier observation. Hence, $(\mathfrak{B}, \phi) \vDash \mathrm{UF}$.

Remark. As a corollary of the proof, every infinite set definable in $(\mathfrak{B}, A)$ has cardinality that of $A$ or $B$. 
In summary, all of the following are preserved in passing from $T$ to $T^{\mathrm{t}}$ : completeness, constructibility of definable sets (hence also NDG), local o-minimality, and UF. We shall not attempt in this paper to analyze $T^{\mathrm{t}}$ any further.

Remark. After appropriate modification (another unary predicate is needed) and further work, results similar to those from [12] hold for complete o-minimal theories extending the theory of densely ordered groups. See Lewenberg [22] for details.

A nonstandard linear continuum. By $[28$, every expansion of $(\mathbb{R},<)$ that defines no countably infinite unary sets has o-minimal open core. The proof given there depends on working over $\mathbb{R}$. A natural question is whether there are useful generalizations of this result to other linear continua. We now cast some doubt on this.

Consider $R:=\mathbb{R} \times[-1,1] \subseteq \mathbb{R}^{2}$ in the lexicographic order. It is an exercise to see that $(R,<)$ is Dedekind complete. Thus, every expansion of $(R,<)$ satisfies DC. Define $\lambda, \mu, \rho: R \rightarrow R$ by

$$
\begin{aligned}
\lambda((a, b)) & =(a,-1), \\
\mu((a, b)) & =(a, 0), \\
\rho((a, b)) & =(a, 1) .
\end{aligned}
$$

Put $\mathfrak{R}=(R,<, \lambda, \mu, \rho)$. The graph of $\mu$ is locally closed, and both $\lambda$ and $\rho$ are definable in $(R,<, \mu)$. As $\rho(R)=\mathbb{R} \times\{1\}$, it follows that $\mathfrak{R}^{\circ}$ is not even locally o-minimal. Nevertheless, again via $\mathrm{QE}$,

7.5. $\mathfrak{R} \vDash \mathrm{UF}$, every definable set either has interior or is nowhere dense, and every infinite definable set has cardinality that of $R$.

D-minimality. We close this section by pointing out that not even UF is necessary for a certain amount of good behavior. Evidently, $\left(\mathbb{R},+, \cdot, 2^{\mathbb{N}}\right) \not \models$ UF, where $2^{\mathbb{N}}=$ $\left\{2^{n}: n \in \mathbb{N}\right\}$. Yet there is some uniformity in place: given definable $A \subseteq \mathbb{R}^{m+1}$, there exists $N$ such that for every $x \in \mathbb{R}^{m}$ the fiber $A_{x}$ either has interior or is a union of $N$ discrete sets. (This property has been tentatively called "d-minimality", at least, for expansions of the real line.) With a little basic topology, the proof of Definable Choice for o-minimal expansions of ordered groups (recall 1.13) can be elaborated upon so as to hold in this setting [27. Of course, definable Skolem functions and elimination of imaginaries then follow just as in the o-minimal case. More difficult is that a countable cell decomposition theorem holds and, for each $p \in \mathbb{N}$, every definable set is a finite union of $C^{p}$ submanifolds (not required to be connected), each of which is definable [26, §4]. See also [6, 17, 30] for more information and other examples.

\section{Questions}

We close with a list of questions and suggestions for further research.

In Theorem A, can UF be relaxed to assuming only that every discrete definable subset of $R$ is finite? (Recall 2.14.) What if $\Re$ expands an ordered field?

We have shown that the group structure is necessary in Theorem A. What about in Theorem $\mathrm{A}^{\prime}$ ? What if we also assume that $T \vDash \mathrm{EP}$ ?

If $S \subseteq R$ and $(\mathfrak{R}, S)^{\circ}$ is o-minimal, is $(\mathfrak{R}, S)^{\circ}=\mathfrak{R}^{\circ}$ ?

If $\mathrm{DOG} \subseteq T \models \mathrm{DC}+\mathrm{EP}$, does $T \vDash \mathrm{NDG}$ ? What if $T$ extends the theory of ordered fields and has a model over $\mathbb{R}$ ? 
If $\mathfrak{R}$ has definable Skolem functions and $\mathfrak{R}^{\circ}$ is o-minimal, is $\mathfrak{R}$ o-minimal? (Recall 5.4 and 6.3 ) What if $\mathfrak{R}$ expands the real field?

If $\mathfrak{R} \vDash \mathrm{DC}+\mathrm{EP}+\mathrm{NIP}$ and expands an ordered group, is $\mathfrak{R}$ o-minimal? What if $\mathfrak{R}$ expands an ordered field?

Suppose that $\mathfrak{R}$ expands a densely ordered group, has an Archimedean model, and $\mathfrak{R}^{\circ}$ is o-minimal. Does $\operatorname{Th}(\mathfrak{R})$ have a model over $\mathbb{R}$ that is unique up to isomorphism? (Recall that this is true if $\mathfrak{R}$ is o-minimal [21.)

Investigate connections with the emerging subject of rosy theories 2, 31. For example, let $T$ be a complete extension of the theory of ordered fields. It can be shown that if $T \vDash \mathrm{DC}$ and is rosy, then $T \vDash \mathrm{UF}$. By Theorem A, every model of $T$ then has an o-minimal open core. To what extent does a converse hold?

Find a natural mathematical interpretation or application of generic sets for o-minimal expansions $\mathfrak{R}$ of the real field. (Given the properties of generic sets, an argument could be made that "generic for $\mathfrak{R}$ " could be called "random for $\mathfrak{R}$ ".)

Analyze tame pairs (as in Section 7) as extensively as dense pairs.

Let $(K,+, \cdot)$ be a subfield of $(\mathbb{R},+, \cdot)$ that does not define $\mathbb{Z}$. Is $(\mathbb{R},+, \cdot, K)^{\circ}={ }_{\mathrm{df}}$ $(\mathbb{R},+, \cdot)$ ?

Are there reasonable versions of Theorem A for weak or local o-minimality? Results from 4 suggest that the former would require some subtlety.

Let $\mathfrak{M}$ be an expansion of a valued field. It makes sense to define the open core of $\mathfrak{M}$ with respect to the valuation topology. What can be said about $\mathfrak{M}$ and its open core? For example, if $\mathfrak{M}$ expands a $p$-adically closed field, is there a $P$-minimal version of Theorem A? See Haskell and Macpherson [18 for the definition of $P$-minimality and more information.

\section{REFERENCES}

[1] O. Belegradek and B. Zilber, The model theory of the field of reals with a subgroup of the unit circle, J. Lond. Math. Soc. (2) 78 (2008), no. 3, 563-579. MR2456892 (2009i:03029)

[2] A. Berenstein, C. Ealy, and A. Günaydın, Thorn independence in the field of real numbers with a small multiplicative group, Ann. Pure Appl. Logic 150 (2007), no. 1-3, 1-18. MR2370512

[3] Z. Chatzidakis and A. Pillay, Generic structures and simple theories, Ann. Pure Appl. Logic 95 (1998), no. 1-3, 71-92. MR1650667 (2000c:03028)

[4] A. Dolich, A note on weakly o-minimal structures and definable completeness, Notre Dame J. Formal Logic 48 (2007), no. 2, 281-292 (electronic). MR2306397(2008i:03042)

[5] R. Dougherty and C. Miller, Definable Boolean combinations of open sets are Boolean combinations of open definable sets, Illinois J. Math. 45 (2001), no. 4, 1347-1350. MR 1895461 (2003c:54018)

[6] L. van den Dries, The field of reals with a predicate for the powers of two, Manuscripta Math. 54 (1985), no. 1-2, 187-195. MR808687 (87d:03098)

[7] _ o-minimal structures, Logic: from foundations to applications (Staffordshire, 1993), Oxford Sci. Publ., Oxford Univ. Press, New York, 1996, pp. 137-185. MR1428004 (98b:03053)

[8] _ T-convexity and tame extensions. II, J. Symbolic Logic 62 (1997), no. 1, 14-34. MR.1450511 (98h:03048)

[9] , Dense pairs of o-minimal structures, Fund. Math. 157 (1998), no. 1, 61-78. MR1623615 (2000a:03058)

[10] - Tame topology and o-minimal structures, London Mathematical Society Lecture Note Series, vol. 248, Cambridge University Press, Cambridge, 1998. MR.1633348 (99j:03001)

[11] Limit sets in o-minimal structures, Lecture Notes in Real Analytic and Algebraic Geometry, Cuvillier Verlag, Göttingen (2005), 172-215, has appeared in: Proceedings of the RAAG Summer School, Lisbon 2003: O-minimal structures. 
[12] L. van den Dries and A. Lewenberg, T-convexity and tame extensions, J. Symbolic Logic 60 (1995), no. 1, 74-102. MR 1324502 (96a:03048)

[13] L. van den Dries and C. Miller, Geometric categories and o-minimal structures, Duke Math. J. 84 (1996), no. 2, 497-540. MR.1404337(97i:32008)

[14] M. Edmundo, Structure theorems for o-minimal expansions of groups, Ann. Pure Appl. Logic 102 (2000), no. 1-2, 159-181. MR.1732059 (2001k:03080)

[15] S. Fratarcangeli, Elimination of imaginaries in expansions of o-minimal structures by generic sets, J. Symbolic Logic 70 (2005), no. 4, 1150-1160. MR.2194242 (2006i:03061)

[16] H. Friedman, On expansions of o-minimal structures (1996), preliminary report, available at http://www.math.ohio-state.edu/foundations/manuscripts.html

[17] H. Friedman and C. Miller, Expansions of o-minimal structures by fast sequences, J. Symbolic Logic 70 (2005), no. 2, 410-418. MR2140038 (2006a:03053)

[18] D. Haskell and D. Macpherson, A version of o-minimality for the p-adics, J. Symbolic Logic 62 (1997), no. 4, 1075-1092. MR1618009 (99j:03028)

[19] J. Knight, A. Pillay, and C. Steinhorn, Definable sets in ordered structures. II, Trans. Amer. Math. Soc. 295 (1986), no. 2, 593-605. MR833698 (88b:03050b)

[20] M. Laskowski, Vapnik-Chervonenkis classes of definable sets, J. London Math. Soc. (2) 45 (1992), no. 2, 377-384. MR1171563 (93d:03039)

[21] M. Laskowski and C. Steinhorn, On o-minimal expansions of Archimedean ordered groups, J. Symbolic Logic 60 (1995), no. 3, 817-831. MR.1348995 (96i:03032)

[22] A. Lewenberg, On elementary pairs of o-minimal structures, Ph.D. thesis, University of Illinois at Urbana-Champaign, 1995.

[23] A. Macintyre, Dense embeddings. I. A theorem of Robinson in a general setting, Model theory and algebra (A memorial tribute to Abraham Robinson), Lecture Notes in Math., Vol. 498, Springer, Berlin, 1975, pp. 200-219. MR0406788 (53:10574)

[24] D. Macpherson, D. Marker, and C. Steinhorn, Weakly o-minimal structures and real closed fields, Trans. Amer. Math. Soc. 352 (2000), no. 12, 5435-5483 (electronic). MR.1781273 (2001i:03079)

[25] C. Miller, Expansions of dense linear orders with the intermediate value property, J. Symbolic Logic 66 (2001), no. 4, 1783-1790. MR1877021 (2003j:03044)

[26] _ Tameness in expansions of the real field, Logic Colloquium '01, Lect. Notes Log., vol. 20, Assoc. Symbol. Logic, Urbana, IL, 2005, pp. 281-316. MR2143901 (2006j:03049)

[27] _ Definable choice in d-minimal expansions of ordered groups, preliminary report, available at http://www.math.ohio-state.edu/ ${ }^{\sim m i l l e r}$

[28] C. Miller and P. Speissegger, Expansions of the real line by open sets: o-minimality and open cores, Fund. Math. 162 (1999), no. 3, 193-208. MR.1736360 (2001a:03083)

[29] C. Miller and S. Starchenko, A growth dichotomy for o-minimal expansions of ordered groups, Trans. Amer. Math. Soc. 350 (1998), no. 9, 3505-3521. MR.1491870 (99e:03025)

[30] C. Miller and J. Tyne, Expansions of o-minimal structures by iteration sequences, Notre Dame J. Formal Logic 47 (2006), no. 1, 93-99 (electronic). MR2211185 (2006m:03065)

[31] A. Onshuus, Properties and consequences of thorn-independence, J. Symbolic Logic 71 (2006), no. 1, 1-21. MR 2210053 (2007f:03047)

[32] Y. Peterzil and S. Starchenko, A trichotomy theorem for o-minimal structures, Proc. London Math. Soc. (3) 77 (1998), no. 3, 481-523. MR.1643405 (2000b:03123)

[33] Y. Peterzil and C. Steinhorn, Definable compactness and definable subgroups of o-minimal groups, J. London Math. Soc. (2) 59 (1999), no. 3, 769-786. MR.1709079 (2000i:03055)

[34] A. Pillay and C. Steinhorn, Definable sets in ordered structures, Bull. Amer. Math. Soc. (N.S.) 11 (1984), no. 1, 159-162. MR741730 (86c:03033)

[35] _ Definable sets in ordered structures. I, Trans. Amer. Math. Soc. 295 (1986), no. 2, 565-592. MR833697 (88b:03050a)

[36] — Discrete o-minimal structures, Stability in model theory (Trento, 1984), Ann. Pure Appl. Logic 34 (1987), no. 3, 275-289. MR899083 (88j:03023)

[37] _ Definable sets in ordered structures. III, Trans. Amer. Math. Soc. 309 (1988), no. 2, 469-476. MR943306 (89i:03059)

[38] S. Randriambololona, o-minimal structures: Low arity versus generation, Illinois J. Math. 49 (2005), no. 2, 547-558 (electronic). MR2164352 (2006f:03066)

[39] A. Robinson, Solution of a problem of Tarski, Fund. Math. 47 (1959), 179-204. MR0112841 $(22: 3690)$ 
[40] C. Toffalori and K. Vozoris, Notes on local o-minimality (2008), to appear in MLQ Math. Log. Q., available at http://www.logique.jussieu.fr/modnet/Home/

[41] B. Zilber, Complex roots of unity on the real plane (2003), preprint, available at http://www. maths.ox.ac.uk/ zilber/publ.html

Department of Mathematics and Computer Science, Chicago State University, Chicago, Illinois 60628

E-mail address: adolich@csu.edu

Current address: Department of Mathematics, East Stroudsberg University, East Stroudsberg, Pennsylvania 18301

Department of Mathematics, 231 West 18th Avenue, The Ohio State University, Columbus, Ohio 43210

E-mail address: miller@math.ohio-state.edu

URL: http://www.math.ohio-state.edu/ ${ }^{\sim}$ miller

Department of Mathematics, Vassar College, Poughkeepsie, New York 12604

E-mail address: steinhorn@vassar.edu

URL: http://www.math.vassar.edu/faculty/steinhorn 